\title{
GEOMETRIC ISOMORPHISMS BETWEEN INFINITE DIMENSIONAL TEICHMÜLLER SPACES
}

\author{
CLIFFORD J. EARLE AND FREDERICK P. GARDINER
}

\begin{abstract}
Let $X$ and $Y$ be the interiors of bordered Riemann surfaces with finitely generated fundamental groups and nonempty borders. We prove that every holomorphic isomorphism of the Teichmüller space of $X$ onto the Teichmüller space of $Y$ is induced by a quasiconformal homeomorphism of $X$ onto $Y$. These Teichmüller spaces are not finite dimensional and their groups of holomorphic automorphisms do not act properly discontinuously, so the proof presents difficulties not present in the classical case. To overcome them we study weak continuity properties of isometries of the tangent spaces to Teichmüller space and special properties of Teichmüller disks.
\end{abstract}

\section{INTRODUCTION}

Let Teich $(X)$ and Teich $(Y)$ be the Teichmüller spaces of the Riemann surfaces $X$ and $Y$. In this paper we shall study the set of holomorphic isomorphisms of Teich $(X)$ onto itself or onto Teich $(Y)$.

The problem of determining the holomorphic automorphisms of Teich $(X)$ when the Riemann surface $X$ is compact was solved in Royden's fundamental paper [Ro]. Shortly thereafter, in [EK1], Royden's methods were used to find the holomorphic automorphisms of Teich $(X)$ when $X$ is equivalent to the complement of a finite subset of a compact Riemann surface (see also Chapter 9 of [G2]). New methods are needed to find the holomorphic automorphisms of Teich $(X)$ for other Riemann surfaces $X$ because their Teichmüller spaces are infinite dimensional. Until now none of their automorphism groups had been determined.

Our Theorems 1, 2, and 3 determine the holomorphic automorphisms of the Teichmüller spaces of a wide new class of Riemann surfaces. Theorem 4 shows that our class includes all Riemann surfaces with finitely generated fundamental groups and nonempty borders (including all finitely connected bounded plane regions). Theorem 5 classifies the Teichmüller spaces of these surfaces, and Theorem 6 describes all holomorphic isomorphisms between them.

In a forthcoming paper [La], Nikola Lakic extends Theorem 4 to the set of all Riemann surfaces of finite genus with either nonempty border or infinitely generated fundamental group. (That set includes for example the complement of a Cantor set in the plane.) It follows that these surfaces belong to our class, and that Theorems 5 and 6 can be extended to them (see the remark after the statement of Theorem $4)$.

Received by the editors March 15, 1995.

1991 Mathematics Subject Classification. Primary 32G15; Secondary 30C62, 30C75.

Research of the first author was partly supported by NSF Grant DMS 9206924 and by a grant from MSRI; of the second, by NSF Grant DMS 9204069. 
The simplest way to explain our results is to outline the contents of Royden's paper [Ro], calling attention to the new difficulties that arise when Teich $(X)$ is infinite dimensional. The undefined terms in the following discussion will be explained more fully in $\S \S 3$ and 4 of this paper.

A quasiconformal map between two Riemann surfaces induces a holomorphic isomorphism between their Teichmüller spaces; we shall call such isomorphisms geometric. In [Ro] Royden proved that all holomorphic automorphisms of the Teichmüller space of a compact Riemann surface of genus at least two are geometric. Consider a holomorphic automorphism $F$. Royden's proof that $F$ is geometric has three principal steps. Step one is to show that $F$ is an isometry with respect to the Teichmüller metric. To prove this Royden shows that the Teichmüller metric on any of these spaces equals its Kobayashi metric. That statement is of independent interest. It was generalized by Gardiner in [G1], where he showed that the Teichmüller and Kobayashi metrics coincide on the Teichmüller space of any Riemann surface. A new proof based on the theory of holomorphic motions has recently been given in $[\mathrm{EKK}]$.

By step one, the automorphism $F$ induces complex linear isometries between the cotangent spaces at corresponding points $\tau$ and $F(\tau)$. For the Teichmüller spaces under consideration these cotangent spaces can be identified isometrically with the spaces of integrable holomorphic quadratic differentials on the corresponding compact Riemann surfaces. Step two is to study the isometries between these spaces. Royden shows that the existence of such an isometry implies that the corresponding Riemann surfaces are isomorphic. In fact he proves that every compact Riemann surface of genus at least two has the following property, which we shall formalize with a definition.

Definition. Let $Q(X)$ be the complex Banach space of integrable holomorphic quadratic differentials on an arbitrary Riemann surface $X$. We say that $X$ has the isometry property if for any Riemann surfaces $Y$ and $W$ that are quasiconformally equivalent to $X$ every $\mathbb{C}$-linear isometry of $Q(Y)$ onto $Q(W)$ has the form $\varphi \mapsto$ $\alpha f^{*}(\varphi)$, where $\alpha \in \mathbb{C}, f$ is a holomorphic isomorphism of $W$ onto $Y$, and $f^{*}$ is the pull-back mapping induced by $f$.

The final step in Royden's argument uses the isometry property to conclude that the automorphism $F$ is geometric. The main theorem of this paper draws the same conclusion in the general case.

Theorem 1 (the automorphism theorem). Let Teich $(X)$ be the Teichmüller space of an arbitrary Riemann surface $X$. If $X$ has the isometry property, then every holomorphic automorphism of Teich $(X)$ is geometric.

Remark. If $X$ fails to have the isometry property and Teich $(X)$ is finite dimensional, then Teich $(X)$ has non-geometric holomorphic automorphisms. This occurs when $X$ is the sphere with four punctures or a torus with at most two punctures. We do not know whether the converse of Theorem 1 holds when Teich $(X)$ is infinite dimensional. Nor do we know of any Riemann surface that has an infinite dimensional Teichmüller space and fails to have the isometry property.

The automorphism theorem has the following simple proof when Teich $(X)$ is finite dimensional. Any automorphism $F$ preserves Teichmüller distances, and the isometry property implies that the Riemann surfaces corresponding to $\tau$ and $F(\tau)$ 
are isomorphic for each $\tau$ in Teich $(X)$. It follows that for each $\tau$ there is a geometric automorphism $g_{\tau}$ with $F(\tau)=g_{\tau}(\tau)$. Since the group of geometric automorphisms of Teich $(X)$ acts properly discontinuously, $g_{\tau}$ is independent of $\tau$ and $F$ is geometric.

There are two obstacles to using that simple argument in the infinite dimensional case. First, if $X$ is the Riemann surface corresponding to a point $\tau$ in Teichmüller space, the tangent space at $\tau$ is isometric to the dual space $Q^{*}(X)$ of $Q(X)$. When $Q(X)$ is infinite dimensional it is not reflexive (see $\S 6$ ), so it is necessary to prove that an isometry between $Q^{*}(X)$ and $Q^{*}(Y)$ induces an isometry between $Q(Y)$ and $Q(X)$. That is the content of our second theorem.

Theorem 2 (the adjointness theorem). Let $X$ and $Y$ be arbitrary Riemann surfaces. Given any invertible $\mathbb{C}$-linear isometry $T: Q^{*}(X) \rightarrow Q^{*}(Y)$, there is an invertible $\mathbb{C}$-linear isometry $S: Q(Y) \rightarrow Q(X)$ such that $S^{*}=T$.

Note that the adjointness theorem does not require $X$ or $Y$ to have the isometry property. It holds for all Riemann surfaces. Our proof uses properties of an interesting subspace of $Q^{*}(X)$, which is a predual of $Q(X)$ (see $\S 6$ ).

The second obstacle presented by the infinite dimensional case is that the group of geometric automorphisms of Teich $(X)$ does not in general act discontinuously. For example, the Teichmüller space of the unit disk has a transitive group of geometric automorphisms (see $\S 2$ ). However, the adjointness theorem and the isometry property reduce the proof of Theorem 1 to a problem about automorphisms of Teich $(X)$ that fix a point. That problem is solved by our third theorem.

Theorem 3 (the uniqueness theorem). Suppose the Riemann surface $X$ has the isometry property. Let $F$ be a holomorphic automorphism of Teich $(X)$. If $F$ fixes a point $\tau$ of Teich $(X)$ and $F^{\prime}(\tau)$ is a scalar multiple of the identity, then $F$ is the identity map.

Because of the Cartan uniqueness theorem (see Section 8 of $[\mathrm{H}]$ ) the essential step in the proof of Theorem 3 is showing that $F^{\prime}(\tau)$ is the identity. For this purpose we study the effect of $F$ on Teichmüller disks and prove the existence of Teichmüller disks that are not invariant under too many geometric automorphisms (see $\S \S 10-15)$. We also make essential use of the assumption that $X$ has the isometry property. Some restriction on $X$ is certainly needed since the uniqueness theorem fails when Teich $(X)$ is one-dimensional (and therefore isomorphic to the open unit disk).

We shall derive the automorphism theorem from the adjointness and uniqueness theorems in $\S 5$. These theorems will be proved in $\S \S 6-15$.

Our principal remaining theorems apply the automorphism and adjointness theorems to an important special case. We say the Riemann surface $X$ has finite topological type if its fundamental group is finitely generated. Equivalently, there exist a compact Riemann surface $W$ and a closed subset $E$ of $W$ such that $X$ is isomorphic to $W \backslash E$ and $E$ has finitely many components, each of which is a point or a closed analytic disk. The boundaries of these disks are the ideal boundary curves of $X$, and the union of these ideal boundary curves is the border of $X$. We shall prove the following theorem in $§ \S 16-18$.

Theorem 4 (the isometry theorem). Let $X$ and $Y$ be Riemann surfaces of finite topological type. Suppose the border of $X$ is not empty. If $S: Q(Y) \rightarrow Q(X)$ is 
a surjective $\mathbb{C}$-linear isometry, there are a complex number $\alpha$ and a holomorphic isomorphism $h: X \rightarrow Y$ such that $|\alpha|=1$ and $S(\varphi)=\alpha h^{*}(\varphi)$ for all $\varphi$ in $Q(Y)$.

Remark. The following two theorems are simple consequences of the automorphism, adjointness, and isometry theorems (see $\$ \S 19$ and 20). Lakic's extension of Theorem 4 to the set of all Riemann surfaces of finite genus with either nonempty border or infinitely generated fundamental group therefore extends Theorems 5 and 6 to this important class of surfaces (see [La] for details).

Theorem 5 (the classification theorem). Let $X$ and $Y$ be Riemann surfaces that satisfy the hypotheses of Theorem 4. If there is a complex linear isometry from a single fiber of the tangent bundle to Teich $(X)$ onto some fiber of the tangent bundle to Teich $(Y)$, then there is a quasiconformal mapping from $X$ onto $Y$. In particular there is a geometric isomorphism of Teich $(X)$ onto Teich $(Y)$.

Theorem 6 (the isomorphism theorem). If the Riemann surfaces $X$ and $Y$ satisfy the hypotheses of Theorem 4, then every holomorphic isomorphism of Teich $(X)$ onto Teich $(Y)$ is geometric. In particular, every holomorphic automorphism of Teich $(X)$ is geometric.

We emphasize that the Riemann surfaces in Theorems 4, 5, and 6 are not assumed to be homeomorphic. Let $X$ and $Y$ have finite topological type and nonempty borders, and suppose there is no quasiconformal homeomorphism of $X$ onto $Y$. Then the isomorphism theorem implies that Teich $(X)$ and Teich $(Y)$ are not isomorphic complex manifolds. The classification theorem implies even more: no nonempty open subset of Teich $(X)$ can be mapped into Teich $(Y)$ by a holomorphic map that preserves Teichmüller distances. Analogous results about finite dimensional Teichmüller spaces are well known (see [EK2]), but these are the first such results about the infinite dimensional case.

Some related results about surfaces of infinite genus have recently been obtained by $\mathrm{T}$. Ohsawa. Using different methods he has defined a collection of Riemann surfaces of infinite genus whose Teichmüller spaces are not isomorphic complex manifolds (see $[\mathrm{O}]$ ).

We owe thanks to a number of people and places. J. Milne Anderson provided some stimulating and enlightening conversations and pointed out to us Ng's paper $[\mathrm{Ng}]$. Dragan Vukotić called Kolaski's paper [Ko] to our attention. In addition to support from the NSF, the first author received support and hospitality from MSRI and the Mathematics Institute of the University of Warwick and hospitality from the University of California at Davis. The second author received support while visiting IHES at Bures-sur-Yvette.

\section{The universal Teichmüller Space}

The most important Riemann surface with finite topological type and nonempty border is the open unit disk $\Delta$. Its Teichmüller space Teich $(\Delta)$ is often called the universal Teichmüller space. We shall begin with a brief discussion of that space, since it provides the most concrete example to which our theorems apply.

Let $S^{1}$ be the unit circle in the complex plane $\mathbb{C}$. By definition, an orientationpreserving homeomorphism of $S^{1}$ onto itself is quasisymmetric if it extends to a quasiconformal homeomorphism of the Riemann sphere that maps $\Delta$ onto itself. 
These quasisymmetric homeomorphisms form a group $Q S$. The group $G$ of holomorphic automorphisms of $\Delta$ consists of Möbius transformations, and we regard $G$ as a subgroup of $Q S$ by identifying each $g$ in $G$ with its restriction to $S^{1}$. The set $G \backslash Q S$ of right cosets of $G$ in $Q S$ is, by definition, the universal Teichmüller space $\operatorname{Teich}(\Delta)$.

For each $f$ in $Q S$, right translation by $f^{-1}$ induces a bijective map $\rho_{f}$ of Teich $(\Delta)$ onto itself: $\rho_{f}(G h)=G h f^{-1}$. By definition, the geometric automorphisms of $\operatorname{Teich}(\Delta)$ are the maps $\rho_{f}, f$ in $Q S$. Observe that the group of geometric automorphisms acts transitively on $\operatorname{Teich}(\Delta)$.

It is well known that the geometric automorphisms are holomorphic with respect to the natural complex manifold structure on Teich $(\Delta)$ (see [G2] or [Na]). The original goal of our research was to prove the converse statement, that all holomorphic automorphisms of Teich $(\Delta)$ are geometric. That result is a special case of the automorphism theorem, because $\Delta$ has the isometry property. In fact, every Riemann surface that is quasiconformally equivalent to $\Delta$ is isomorphic to $\Delta$, so the isometry property for $\Delta$ states only that every $\mathbb{C}$-linear isometry of $Q(\Delta)$ onto itself has the form $\varphi \mapsto \alpha h^{*}(\varphi)$ for some $\alpha$ in $\mathbb{C}$ and some automorphism $h$ of $\Delta$. That statement is a special case of a theorem of Kolaski [Ko], since $Q(\Delta)$ is isometrically isomorphic to the space of integrable holomorphic functions on $\Delta$, with its usual $L^{1}$ norm.

\section{TeichmülleR SPACE AND ITS TANGENT BUNDLE}

In $\S \S 3$ and 4 we shall review some well known facts about Teichmüller spaces, starting with some definitions. The border $\partial X$ of a Riemann surface $X$ is the union of its ideal boundary curves (see [G2]). Any quasiconformal homeomorphism $f$ of $X$ onto a Riemann surface $Y$ extends uniquely to a homeomorphism of $X \cup \partial X$ onto $Y \cup \partial Y$. For two such qc homeomorphisms $f_{1}$ and $f_{2}$ from $X$ onto the Riemann surfaces $Y_{1}$ and $Y_{2}$, respectively, we say that $f_{1}$ and $f_{2}$ are equivalent if there is a holomorphic isomorphism $g$ of $Y_{1}$ onto $Y_{2}$ such that the homeomorphism $f_{2}^{-1} \circ g \circ f_{1}$ of $X \cup \partial X$ onto itself is homotopic to the identity by a homotopy that maps $X$ into itself and fixes $\partial X$ pointwise. By definition, the Teichmüller space Teich $(X)$ of $X$ is the set of all qc homeomorphisms with domain $X$ factored by this equivalence relation. We denote the equivalence class of the qc homeomorphism $f: X \rightarrow Y$ by $[f]$.

For any qc homeomorphism $g$ of $X$ onto $Y$, right translation by $g^{-1}$ induces a bijective map $\rho_{g}$ from Teich $(X)$ to Teich $(Y): \rho_{g}([f])=\left[f \circ g^{-1}\right]$. We call the bijection $\rho_{g}$ a geometric isomorphism.

The basepoint of $\operatorname{Teich}(X)$ is the equivalence class of the identity map $i d: X \rightarrow$ $X$. If $g$ is a qc homeomorphism of $X$ onto $Y$, then $\rho_{g}$ maps the point $[g]$ of Teich $(X)$ to the basepoint of $T \operatorname{eich}(Y)$.

A fundamental theorem about Teichmüller spaces states that they have complex manifold structures such that all geometric isomorphisms are holomorphic (see [G2] or $[\mathrm{Na}]$ ). To describe that complex structure we first introduce two Banach spaces of tensors on the Riemann surface $X$. By definition, a quadratic differential $\varphi$ on $X$ is a tensor whose restriction to the domain $U$ of any local coordinate $z$ has the form $f d z^{2}$, where $f$ is a measurable function on $U$. We say that $\varphi$ is holomorphic (or meromorphic) if all these functions $f$ are holomorphic (or meromorphic).

If $\varphi$ is a quadratic differential on $X$, we define $|\varphi|$ to be the differential 2-form 
on $X$ whose restriction to $U$ equals $|f| d x d y$ if $\varphi$ equals $f d z^{2}$ in $U$. We say $\varphi$ is integrable if its norm

$$
\|\varphi\|=\iint_{X}|\varphi|
$$

is finite. The space $Q(X)$ of all integrable holomorphic quadratic differentials on $X$ is a complex Banach space with the norm (3.1).

A Beltrami form on $X$ is a tensor $\mu$ whose restriction to the domain $U$ of any local coordinate $z$ has the form $f d \bar{z} / d z$, where $f$ is a bounded measurable function on $U$. The norm $\|\mu\|_{\infty}$ is defined as the least upper bound of the $L^{\infty}$ norms of all these functions $f$. We denote by $\operatorname{Belt}(X)$ the complex Banach space of Beltrami forms of finite norm.

If $\mu \in \operatorname{Belt}(X)$ and $\varphi \in Q(X)$, we define $\mu \varphi$ to be the differential 2-form on $X$ that equals $f g d x d y$ in $U$ if $\mu$ equals $f d \bar{z} / d z$ and $\varphi$ equals $g d z^{2}$ in $U$. If $\varphi$ is not identically zero, there is a unique $\mu$ in $\operatorname{Belt}(X)$ such that $\mu \varphi=|\varphi|$. We denote it by $|\varphi| / \varphi$.

The natural pairing

$$
(\mu, \varphi)_{X}=\iint_{X} \mu \varphi, \quad \mu \in \operatorname{Belt}(X), \varphi \in Q(X),
$$

between $\operatorname{Belt}(X)$ and $Q(X)$ induces a linear map $P$ of $\operatorname{Belt}(X)$ onto $Q^{*}(X)$, the dual space of $Q(X)$, defined by

$$
P \mu(\varphi)=(\mu, \varphi)_{X} \quad \text { if } \mu \in \operatorname{Belt}(X) \text { and } \varphi \in Q(X) .
$$

The kernel of $P$ is $Q(X)^{\perp}=\left\{\mu \in \operatorname{Belt}(X) ;(\mu, \varphi)_{X}=0\right.$ for all $\left.\varphi \in Q(X)\right\}$.

Let $M(X)$ be the open unit ball of $\operatorname{Belt}(X)$. By definition, the Beltrami coefficient of the quasiconformal homeomorphism $f: X \rightarrow Y$ is the Beltrami form $\mu_{f}$ in $M(X)$ that equals $\bar{\partial}(z \circ f) / \partial(z \circ f)$ in $f^{-1}(U)$ whenever $z$ is a local coordinate defined on an open set $U \subset Y$. Every $\mu$ in $M(X)$ is the Beltrami coefficient of a qc homeomorphism. In fact let $X^{\mu}$ be the Riemann surface whose underlying topological space is the underlying topological space of $X$ and whose local coordinates are the homeomorphisms $w$ from open subsets of $X$ onto open subsets of $\mathbb{C}$ such that $\mu=\bar{\partial} w / \partial w$ in the domain of $w$. The identity map from $X$ to $X^{\mu}$ is a qc homeomorphism whose Beltrami coefficient is $\mu$.

Define the map $\Phi: M(X) \rightarrow \operatorname{Teich}(X)$ by $\Phi(\mu)=\left[i d: X \rightarrow X^{\mu}\right]$. That map is surjective. Since it depends on the Riemann surface $X$, we shall occasionally denote it by $\Phi_{X}$ to avoid ambiguity. Note that $X^{0}=X$, so $\Phi(0)$ is the basepoint of $\operatorname{Teich}(X)$.

The complex manifold structure of Teich $(X)$ is determined by the property that $\Phi$ is a holomorphic map whose Fréchet derivative at any point of $M(X)$ has a continuous right inverse (see [G2] or $[\mathrm{Na}]$ ). Therefore the tangent space to Teich $(X)$ at $\Phi(0)$ is isomorphic to the quotient space $\operatorname{Belt}(X) / \operatorname{Ker}\left(\Phi^{\prime}(0)\right)$. Since the kernel of $\Phi^{\prime}(0)$ is precisely $Q(X)^{\perp}$ (see [G2] or [Na]), the map

$$
\Phi^{\prime}(0) \mu \mapsto P \mu
$$

identifies that quotient space with the space $Q^{*}(X)$. That gives us a natural identification between $Q^{*}(X)$ and the tangent space to Teich $(X)$ at its basepoint. To understand the tangent space at $[g]$ we can use the geometric isomorphism $\rho_{g}$ to identify the tangent space to Teich $(X)$ at $[g]$ with the tangent space to Teich $(g(X))$ at its basepoint. 


\section{IsOMETRIES OF THE TANGENT SPACE}

It is well known that the Teichmüller metric $d$ on Teich $(X)$ is induced by a Finsler structure on its tangent bundle (see Chapter 7 of [G2]). That Finsler structure defines a norm, called the Teichmüller norm, on the tangent space at each point of Teich $(X)$. The Teichmüller norm on the tangent space at the basepoint is given by

$$
\left\|\Phi^{\prime}(0) \mu\right\|=\lim _{t \rightarrow 0^{+}} \frac{d(\Phi(0), \Phi(t \mu))}{t}=\sup \left\{\left|(\mu, \varphi)_{X}\right| ; \varphi \in A(X) \text { and }\|\varphi\|=1\right\}
$$

(see [G2]). The supremum on the right hand side is precisely the norm of $P \mu$ in $Q^{*}(X)$. Thus, under the identification (3.3), the Teichmüller norm coincides with the standard dual norm on $Q^{*}(X)$. This leads immediately to

Lemma 4.1. Let $X$ and $Y$ be Riemann surfaces. If $F:$ Teich $(X) \rightarrow$ Teich $(Y)$ is a holomorphic isomorphism that preserves basepoints, then $F^{\prime}([i d]): Q^{*}(X) \rightarrow Q^{*}(Y)$ is an invertible $\mathbb{C}$-linear isometry.

Proof. We are using (3.3) to identify the tangent spaces of Teich $(X)$ and Teich $(Y)$ at their basepoints with $Q^{*}(X)$ and $Q^{*}(Y)$ respectively. Since $F$ and $F^{-1}$ are holomorphic, $F^{\prime}([i d])$ is an invertible $\mathbb{C}$-linear map. It preserves Teichmüller norms because the Teichmüller metrics on Teich $(X)$ and Teich $(Y)$ coincide with their respective Kobayashi metrics (see [G1]), and any holomorphic isomorphism preserves Kobayashi distances.

The geometric isomorphism $\rho_{f}$ :Teich $(X) \rightarrow$ Teich $(Y)$ induced by a holomorphic isomorphism $f: X \rightarrow Y$ satisfies the hypotheses of Lemma 4.1, and we shall need to understand the isometry $\rho_{f}^{\prime}([i d])$. For that purpose we introduce the pullbacks of quadratic differentials and Beltrami forms induced by a holomorphic map $f: X \rightarrow Y$. If $f$ is such a map and $\varphi$ is a quadratic differential on $Y$, then $f^{*}(\varphi)$ is the quadratic differential on $X$ that equals $(g \circ f)(d(z \circ f))^{2}$ in $f^{-1}(U)$ whenever $\varphi$ equals $g d z^{2}$ in $U \subset Y$ and $z$ is a local coordinate defined on $U$. If $f$ is not constant and $\mu$ is a Beltrami form on $Y$, then $f^{*}(\mu)$ is the Beltrami form on $X$ that equals $(g \circ f) d(\bar{z} \circ f) / d(z \circ f)$ in $f^{-1}(U)$ whenever $\mu$ equals $g d \bar{z} / d z$ in $U$ and $z$ is a local coordinate defined on $U$.

When $f: X \rightarrow Y$ is a holomorphic isomorphism, the maps $S_{f}(\varphi)=f^{*}(\varphi)$ and $T_{f}(\mu)=f^{*}(\mu)$ are invertible $\mathbb{C}$-linear isometries of $Q(Y)$ onto $Q(X)$ and $\operatorname{Belt}(Y)$ onto $\operatorname{Belt}(X)$ respectively. It is obvious that

$$
\left(T_{f}(\mu), S_{f}(\varphi)\right)_{X}=(\mu, \varphi)_{Y} \quad \text { for all } \mu \in \operatorname{Belt}(Y) \text { and } \varphi \in Q(Y) \text {. }
$$

Lemma 4.2. Let $X$ and $Y$ be Riemann surfaces, $f$ a holomorphic isomorphism of $X$ onto $Y$, and $\rho_{f}:$ Teich $(X) \rightarrow$ Teich $(Y)$ the induced geometric isomorphism of Teichmüller spaces. Then $\rho_{f}^{\prime}([i d]): Q^{*}(X) \rightarrow Q^{*}(Y)$ is the adjoint of $S_{f}$.

Proof. The definition of $T_{f}$ makes $f: X^{T_{f}(\mu)} \rightarrow Y^{\mu}$ a holomorphic isomorphism for every $\mu$ in $M(Y)$. Therefore

$\rho_{f}\left(\Phi_{X}\left(T_{f}(\mu)\right)\right)=\left[f^{-1}: Y \rightarrow X^{T_{f}(\mu)}\right]=\left[i d: Y \rightarrow Y^{\mu}\right]=\Phi_{Y}(\mu)$ for all $\mu \in M(Y)$. 
Differentiation at $\mu=0$ gives $\rho_{f}^{\prime}([i d]) \circ \Phi_{X}{ }^{\prime}(0) \circ T_{f}=\Phi_{Y}{ }^{\prime}(0)$. Thus $\rho_{f}{ }^{\prime}([i d])$ corresponds via (3.3) to the linear map $T$ that sends $P_{X}\left(T_{f}(\mu)\right)$ to $P_{Y} \mu$ for each $\mu$ in $\operatorname{Belt}(Y)$. It follows immediately from (4.1) that $T$ is the adjoint of $S_{f}$.

Remark. The fact that the isometries $\rho_{f}^{\prime}([i d])$ in Lemma 4.2 are adjoints of isometries of $Q(Y)$ onto $Q(X)$ was predicted by the adjointness theorem.

\section{Proof of the automorphism theorem}

In this section we deduce the automorphism theorem from the adjointness and uniqueness theorems. First we prove the following lemma, which is a consequence of Lemmas 4.1 and 4.2 and the adjointness theorem.

Lemma 5.1. Let $X$ be a Riemann surface with the isometry property, and let $F$ be a holomorphic automorphism of Teich $(X)$. For each $\tau$ in Teich $(X)$ there is a geometric automorphism $g_{\tau}$ of Teich $(X)$ such that $F(\tau)=g_{\tau}(\tau)$ and $F^{\prime}(\tau)=$ $\alpha_{\tau} g_{\tau}{ }^{\prime}(\tau)$ for some complex number $\alpha_{\tau}$.

Proof. Choose quasiconformal homeomorphisms $g: X \rightarrow W$ and $h: X \rightarrow Y$ so that $\tau=[g]$ and $F(\tau)=[h]$. Put $\widetilde{F}=\rho_{h} \circ F \circ \rho_{g}{ }^{-1}: \operatorname{Teich}(W) \rightarrow T e i c h(Y)$. Since $\widetilde{F}$ is a holomorphic isomorphism that preserves basepoints, Lemma 4.1 implies that $\widetilde{F}^{\prime}([i d])$ is a $\mathbb{C}$-linear isometry of $Q^{*}(W)$ onto $Q^{*}(Y)$. By the adjointness theorem there is a $\mathbb{C}$-linear isometry $S$ of $Q(Y)$ onto $Q(W)$ such that $\widetilde{F}^{\prime}([i d])=S^{*}$. By the hypothesis that $X$ has the isometry property, there exist a complex number $\alpha$ and a holomorphic isomorphism $f$ of $W$ onto $Y$ such that $S(\varphi)=\alpha f^{*}(\varphi)$ for all $\varphi$ in $Q(Y)$. The geometric isomorphism $\rho_{f}:$ Teich $(W) \rightarrow$ Teich $(Y)$ preserves basepoints and, by Lemma 4.2, $\widetilde{F}^{\prime}[i d]=\alpha \rho_{f}^{\prime}[i d]$. We claim that the geometric isomorphism $g_{\tau}=\left(\rho_{h}\right)^{-1} \circ \rho_{f} \circ \rho_{g}\left(=\rho_{h^{-1} \circ f \circ g}\right)$ has the required properties. In fact it is clear that $g_{\tau}(\tau)=F(\tau)$, and the chain rule gives

$$
F^{\prime}(\tau)=\left(\left(\rho_{h}\right)^{-1} \circ \widetilde{F} \circ \rho_{g}\right)^{\prime}(\tau)=\alpha\left(\left(\rho_{h}\right)^{-1} \circ \rho_{f} \circ \rho_{g}\right)^{\prime}(\tau)=\alpha g_{\tau}^{\prime}(\tau) .
$$

The automorphism theorem follows trivially from Lemma 5.1 and the uniqueness theorem. Suppose the Riemann surface $X$ has the isometry property. Let $\widetilde{F}$ be a holomorphic automorphism of Teich $(X)$ and let $\tau$ be any point of Teich $(X)$. Lemma 5.1 gives us a geometric automorphism $g_{\tau}$ of Teich $(X)$ such that $F=$ $\widetilde{F}^{-1} \circ g_{\tau}$ satisfies the hypotheses of the uniqueness theorem. Therefore $F$ is the identity map and $\widetilde{F}$ is the geometric isomorphism $g_{\tau}$.

\section{A DUALity THEOREM}

We shall prove the adjointness theorem in Section 9, after studying some geometric properties of the space $Q^{*}(X)$. It is already known (see [E2] and [E3]) that $Q^{*}(X)$ has a closed subspace $Q^{*}(X)_{0}$ whose dual space is isometrically isomorphic to $Q(X)$. In this section we shall reprove that result by a simpler method that provides a useful new characterization of $Q^{*}(X)_{0}$. The Riemann surface $X$ will be fixed throughout Sections 6,7 , and 8 , so we shall simplify our notation, setting $A=Q(X), B=Q^{*}(X)$, and $B_{0}=Q^{*}(X)_{0}$.

Our first objective is to define the subspace $B_{0}$. For that purpose we introduce on $A$ the compact-open topology of uniform convergence on compact subsets of $X$. The 
standard argument, using the mean value property of holomorphic functions and Montel's theorem, shows that the closed unit ball of $A$ is compact in the compactopen topology and that this topology is determined by the family of continuous seminorms

$$
p_{K}(\varphi)=\iint_{K}|\varphi|, \quad \varphi \in A
$$

where $K$ is a compact subset of $X$. It is metrizable since $X$ is a countable union of compact sets.

Definitions. A sequence $\left(\varphi_{n}\right)$ in $A$ is called a degenerating sequence if $\left\|\varphi_{n}\right\| \leq 1$ for all $n$ and $\varphi_{n} \rightarrow 0$ in the compact-open topology as $n \rightarrow \infty$. (In other words $p_{K}\left(\varphi_{n}\right) \rightarrow 0$ as $n \rightarrow \infty$, for every compact set $K \subset X$.) For each linear functional $v$ in $B$ set

$\beta(v)=\inf \left\{r \in \mathbb{R} ; \limsup _{n \rightarrow \infty}\left|v\left(\varphi_{n}\right)\right| \leq r\right.$ for every degenerating sequence $\left(\varphi_{n}\right)$ in $\left.A\right\}$.

Lemma 6.1. The function $\beta$ is a continuous seminorm on $B$.

Proof. It is clear that $\beta(v+w) \leq \beta(v)+\beta(w)$ and $\beta(c v)=|c| \beta(v)$ for all $v$ and $w$ in $B$ and $c$ in $\mathbb{C}$, so $\beta$ is a seminorm. The obvious inequality $\beta(v) \leq\|v\|$ implies that $\beta$ is continuous, since $|\beta(v)-\beta(w)| \leq \beta(v-w)$ for all $v$ and $w$ in $B$ (see Theorem 1.34 in $[\mathrm{Ru} 2])$.

Corollary and Definition. The set $\{v \in B ; \beta(v)=0\}$ is a closed subspace of $B$, which we shall denote by $B_{0}$.

First we prove an elementary property of $B_{0}$ that will be needed in $\S 9$.

Lemma 6.2. For each $v$ in $B$ there is a sequence $\left(v_{n}\right)$ in $B_{0}$ such that $\left\|v_{n}\right\| \leq\|v\|$ for every $n$ and $v_{n} \rightarrow v$ in the weak $k^{*}$ topology as $n \rightarrow \infty$.

Proof. Let $\mu$ in $\operatorname{Belt}(X)$ satisfy $P \mu=v$ and $\|\mu\|_{\infty}=\|v\|$. Choose an increasing sequence $\left(K_{n}\right)$ of compact subsets of $X$ whose union is $X$ and set

$$
v_{n}(\varphi)=\iint_{K_{n}} \mu \varphi \quad \text { for all } \varphi \in A
$$

The sequence $\left(v_{n}\right)$ has all the required properties.

Next we prove that $B_{0}$ is a predual of $A$.

Lemma 6.3. The map $L$ from $A$ to the dual space $B_{0}^{*}$ of $B_{0}$ defined by

$$
L \varphi(v)=v(\varphi) \quad \text { for all } \varphi \in A \text { and } v \in B_{0}
$$

is a surjective $\mathbb{C}$-linear isometry.

Proof. Let $\Sigma$ be the closed unit ball of $A$. We know that $\Sigma$ is compact in the compact-open topology. In addition, by its definition, $B_{0}$ is precisely the set of linear functionals $v$ in $B$ whose restriction to $\Sigma$ is continuous with respect to the compact-open topology. These two observations imply that the map $L$ is surjective and isometric. (See the proof of Theorem 1 in $[\mathrm{Ng}]$.) 
Corollary. If $A$ is infinite dimensional, it is not reflexive.

Proof. Let $A$ be infinite dimensional. We shall prove that its predual $B_{0}$ is not reflexive. We use a construction we learned from $\mathrm{H}$. Tanigawa, who attributes it to Curt McMullen (see Proposition 2.1 in $[\mathrm{T}]$ ).

Since $A$ is infinite dimensional, its unit sphere is not compact (in the norm topology), so there is a sequence of unit vectors $\left(\varphi_{n}\right)$ with no norm-convergent subsequence. Passing to a subsequence we may assume that $\left(\varphi_{n}\right)$ converges to $\varphi$ in the compact-open topology. Replacing $\varphi_{n}$ by $\left(\varphi_{n}-\varphi\right) /\left\|\varphi_{n}-\varphi\right\|$ we may assume that $\varphi=0$, so $\left(\varphi_{n}\right)$ is a degenerating sequence of unit vectors.

Now choose a sequence of disjoint compact sets $K_{j}$ and an increasing sequence of integers $n_{j}$ so that $\iint_{K_{j}}\left|\varphi_{n_{j}}\right|>1-2^{-j}$ for all $j \geq 1$. The linear functional

$$
v(\varphi)=\sum_{j=1}^{\infty} \iint_{K_{j}} \frac{\left|\varphi_{n_{j}}\right|}{\varphi_{n_{j}}} \varphi
$$

belongs to $B \backslash B_{0}$ since $\|v\|=\beta(v)=1$.

Remarks. When $X=\Delta$ we can identify $A$ isometrically with the closed subspace $A(\Delta)$ of $L^{1}(\Delta)$ spanned by the polynomials, and the dual space $B$ is isomorphic to the space $B(\Delta)$ of holomorphic functions $g$ on $\Delta$ with norm

$$
\|g\|_{*}=\sup \left\{|g(z)|\left(1-|z|^{2}\right)^{2} ; z \in \Delta\right\}<\infty
$$

(see $[\mathrm{B}]$ or $[\mathrm{SW}]$ ). The isomorphism between $B$ and $B(\Delta)$ is induced, as in [SW], by the pairing

$$
(f, g)=\iint_{\Delta} f(z) g(\bar{z})\left(1-|z|^{2}\right)^{2} d x d y, \quad f \in A(\Delta), g \in B(\Delta) .
$$

Under that isomorphism the predual $B_{0}$ corresponds to the closure $B_{0}(\Delta)$ of the polynomials in $B(\Delta)$. Shields and Williams proved in [SW] that $B_{0}(\Delta)$ is a predual of $A(\Delta)$.

The identification of $B$ with $B(\Delta)$ is not isometric. In fact, if $Q(X)$ has dimension greater than one, $\operatorname{Belt}(X)$ has no closed subspace that is mapped isometrically onto $Q^{*}(X)$ by the projection $P$ defined by (3.2) (see $\S 4$ of $[\mathrm{E} 1]$ ).

\section{Extremal differentials And SEquences in $Q(X)$}

The lemmas in this section are elementary and well known. We provide their proofs for the reader's convenience. We use the notation introduced at the beginning of Section 6.

Definition. An extremal differential for $v$ in $B$ is a quadratic differential $\varphi$ in $A$ such that $\|\varphi\|=1$ and $v(\varphi)=\|v\|$.

Lemma 7.1. A nonzero $v$ in $B$ has at most one extremal differential, and if it has one, then $v$ is an extreme point of the closed ball in $B$ with center 0 and radius $\|v\|$.

Proof. We may assume that $\|v\|=1$ and that $\varphi$ is an extremal differential for $v$. Choose $\mu$ in $\operatorname{Belt}(X)$ so that $\|\mu\|_{\infty}=1$ and $P \mu=v$. Then $0=\|\varphi\|-v(\varphi)=$ $\iint_{X}(|\varphi|-\mu \varphi)$, so $\mu=|\varphi| / \varphi$ and

$$
v(\psi)=\iint_{X}\left(\frac{|\varphi|}{\varphi}\right) \psi \quad \text { for all } \psi \in A .
$$


If $\psi$ is also an extremal differential for $v$, the same reasoning shows that $\mu=|\psi| / \psi$. Therefore the meromorphic function $\psi / \varphi=|\psi / \varphi|$ is a non-negative constant. Since $\psi$ and $\varphi$ both have norm one, $\psi=\varphi$.

Next suppose $2 v=v_{1}+v_{2}$, where $\left\|v_{1}\right\| \leq 1$ and $\left\|v_{2}\right\| \leq 1$. Then

$$
2=2 \operatorname{Re}(v(\varphi))=\operatorname{Re}\left(v_{1}(\varphi)\right)+\operatorname{Re}\left(v_{2}(\varphi)\right) \leq 1+\operatorname{Re}\left(v_{2}(\varphi)\right) \leq 2,
$$

so equality holds throughout, and the extremal differential $\varphi$ for $v$ is also extremal for $v_{1}$ and $v_{2}$. Therefore $v_{1}(\psi)$ and $v_{2}(\psi)$ are both equal to the integral in (7.1) for every $\psi$ in $A$, so $v_{1}=v_{2}=v$ and $v$ is an extreme point of the closed unit ball.

We need the following result from functional analysis.

Lemma 7.2. Let $\left(\varphi_{n}\right)$ be a sequence of unit vectors in $A$. If $\varphi_{n} \rightarrow \varphi$ in the compact-open topology as $n \rightarrow \infty$, then

$$
\lim _{n \rightarrow \infty}\left\|\varphi_{n}-\varphi\right\|=1-\|\varphi\| \text {. }
$$

Proof. We thank Nikola Lakic for showing us the following simple proof.

Since $0 \leq\left|\varphi_{n}-\varphi\right|+|\varphi|-\left|\varphi_{n}\right| \leq 2|\varphi|$ and $\|\varphi\| \leq 1$, we can apply Lebesgue's dominated convergence theorem to obtain

$$
\lim _{n \rightarrow \infty}\left(\left\|\varphi_{n}-\varphi\right\|+\|\varphi\|-\left\|\varphi_{n}\right\|\right)=\iint_{X} \lim _{n \rightarrow \infty}\left(\left|\varphi_{n}-\varphi\right|+|\varphi|-\left|\varphi_{n}\right|\right)=0 .
$$

Definition. An extremal sequence for the linear functional $v$ in $B$ is a sequence $\left(\varphi_{n}\right)$ in $A$ such that $\left\|\varphi_{n}\right\|=1$ for each $n$ and $\lim _{n \rightarrow \infty} v\left(\varphi_{n}\right)=\|v\|$. Every $v$ in $B$ has at least one extremal sequence.

Let $W=\{v \in B ; \beta(v)<\|v\|\}$. Lemma 6.1 and the definition of $B_{0}$ imply that $W$ is an open neighborhood of $B_{0} \backslash\{0\}$.

Lemma 7.3. If $v \in W$, then every extremal sequence for $v$ converges (in norm) to an extremal differential for $v$.

Proof. Since $v$ has at most one extremal differential, it is sufficient to prove that every extremal sequence has a subsequence with the required property. We may therefore assume that the given sequence $\left(\varphi_{n}\right)$ converges in the compact-open topology to a quadratic differential $\varphi$ in $A$ with $\|\varphi\| \leq 1$.

By Lemma 7.2 we know that $\left\|\varphi_{n}-\varphi\right\| \rightarrow 1-\|\varphi\|$ as $n \rightarrow \infty$. Suppose $1-\|\varphi\|$ is positive. Put $\psi_{n}=\left(\varphi_{n}-\varphi\right) /\left\|\varphi_{n}-\varphi\right\|$. Since $\left(\psi_{n}\right)$ is a degenerating sequence,

$$
\beta(v) \geq \limsup _{n \rightarrow \infty}\left|v\left(\psi_{n}\right)\right|=\frac{|\|v\|-v(\varphi)|}{1-\|\varphi\|} \geq \frac{\|v\|-|v(\varphi)|}{1-\|\varphi\|} \geq\|v\|
$$

which contradicts our assumption that $v \in W$. We conclude that $\left\|\varphi_{n}-\varphi\right\| \rightarrow 0$ as $n \rightarrow \infty$. Therefore $\|\varphi\|=1, v(\varphi)=\lim _{n \rightarrow \infty} v\left(\varphi_{n}\right)=\|v\|$, and $\varphi$ is extremal for $v$.

Remark. The use of Lemma 7.2 to analyse the degenerating sequence $\left(\psi_{n}\right)$ is a standard method in the theory of extremal quasiconformal mappings. We first encountered it (with $X=\Delta$ ) in the work of Strebel (see [S1] and [S2]). It was formalized by Harrington and Ortel in Propositions 1.1 and 1.2 of [HO], again with $X=\Delta$. 


\section{NON-UNIFORM CONVEXITY OF $Q^{*}(X)_{0}$}

We shall continue to use the notation introduced in Section 6. Lemmas 7.1 and 7.3 imply that $B_{0}\left(=Q^{*}(X)_{0}\right)$ is strictly convex. Unless its dimension is finite $B_{0}$ is not reflexive, so it cannot be uniformly convex. (See p. 410 of [Ru2].) The following lemma, which indicates that uniform convexity fails rather dramatically, is the key to our proof of the adjointness theorem.

Lemma 8.1. Suppose $v \in B_{0}$. Let $\left(w_{n}\right)$ be any sequence in $B$ that converges to zero in the weak* topology. If $\limsup _{n \rightarrow \infty}\left\|w_{n}\right\| \leq\|v\|$, then

$$
\left\|v \pm w_{n}\right\| \rightarrow\|v\| \text { as } n \rightarrow \infty
$$

Proof. We may assume that $\|v\|>0$, since otherwise (8.1) is an immediate consequence of the hypotheses. The sequences $\left( \pm w_{n}\right)$ satisfy the hypotheses of the lemma together, so it suffices to prove that $\left\|v+w_{n}\right\| \rightarrow\|v\|$ as $n \rightarrow \infty$. Since $v+w_{n} \rightarrow v$ in the weak* topology, we know that $\liminf _{n \rightarrow \infty}\left\|v+w_{n}\right\| \geq\|v\|$. We need to prove that $\limsup _{n \rightarrow \infty}\left\|v+w_{n}\right\| \leq\|v\|$.

If the required inequality fails, we can pass to a subsequence so that

$$
\lim _{n \rightarrow \infty}\left\|v+w_{n}\right\|=L>\|v\|
$$

Passing again to a subsequence we may assume that $\left\|w_{n}\right\|<(\|v\|+L) / 2<\left\|v+w_{n}\right\|$ for all $n$. Then $\beta\left(v+w_{n}\right) \leq \beta\left(w_{n}\right) \leq\left\|w_{n}\right\|<\left\|v+w_{n}\right\|$, so $v+w_{n} \in W$ for every $n$. Therefore, by Lemma 7.3, each $v+w_{n}$ has an extremal differential $\varphi_{n}$. Passing if necessary to a further subsequence we may assume that $\varphi_{n}$ converges in the compact-open topology to a quadratic differential $\varphi$ in $A$ as $n \rightarrow \infty$.

Suppose that $\lim _{n \rightarrow \infty}\left\|\varphi_{n}-\varphi\right\|$ (which equals $1-\|\varphi\|$, by Lemma 7.2) is positive. Let $\left(\psi_{n}\right)$ be the degenerating sequence defined in the proof of Lemma 7.3. Then

$$
\limsup _{n \rightarrow \infty}\left|\left(v+w_{n}\right)\left(\psi_{n}\right)\right| \leq \limsup _{n \rightarrow \infty}\left|w_{n}\left(\psi_{n}\right)\right| \leq \limsup _{n \rightarrow \infty}\left\|w_{n}\right\| \leq\|v\| .
$$

But we also have

$$
\limsup _{n \rightarrow \infty}\left|\left(v+w_{n}\right)\left(\psi_{n}\right)\right|=\limsup _{n \rightarrow \infty} \frac{\left|\left\|\left(v+w_{n}\right)\right\|-\left(v+w_{n}\right)(\varphi)\right|}{\left\|\varphi_{n}-\varphi\right\|}=\frac{|L-v(\varphi)|}{1-\|\varphi\|}>\|v\| .
$$

This contradicts (8.3). Therefore $\varphi_{n} \rightarrow \varphi$ in the norm topology of $A$, and

$$
L=\lim _{n \rightarrow \infty}\left\|v+w_{n}\right\|=\left|\lim _{n \rightarrow \infty}\left(v+w_{n}\right)\left(\varphi_{n}\right)\right|=\left|\lim _{n \rightarrow \infty}\left(v+w_{n}\right)(\varphi)\right|=|v(\varphi)| \leq\|v\|<L .
$$

This contradiction shows that (8.2) is impossible.

We shall need the following rather elementary converse of Lemma 8.1 in the next section. 
Lemma 8.2. Let $v$ in $B$ be an extreme point of the closed ball with center 0 and radius $\|v\|$. If $\left(w_{n}\right)$ is a sequence in $B$ such that (8.1) holds, then $\limsup _{n \rightarrow \infty}\left\|w_{n}\right\| \leq\|v\|$ and $w_{n} \rightarrow 0$ in the weak $k^{*}$ topology.

Proof. Obviously $\limsup 2\left\|w_{n}\right\| \leq \lim \sup \left(\left\|w_{n}+v\right\|+\left\|w_{n}-v\right\|\right)=2\|v\|$ by (8.1). This proves the first conclusion of the lemma and implies the existence of a number $C$ such that $\left\|w_{n}\right\| \leq C$ for all $n$. Since the Banach space $A$ is separable, the closed ball in $B$ with center 0 and radius $C$ is sequentially compact in the weak* topology (see Theorems 3.15 and 3.16 in [Ru2]). We may therefore assume with no loss of generality that $w_{n} \rightarrow u\left(\right.$ weak $\left.^{*}\right)$ as $n \rightarrow \infty$. We must prove that $u=0$.

Since $v \pm w_{n} \rightarrow v \pm u$ ( weak $^{*}$ ) as $n \rightarrow \infty$, formula (8.1) implies that

$$
\|v \pm u\| \leq \lim _{n \rightarrow \infty}\left\|v \pm w_{n}\right\|=\|v\|
$$

Therefore the line segment $[v-u, v+u]$ lies in the closed ball with center 0 and radius $\|v\|$. Since $v$ is an extreme point of that ball, $u=0$.

Remark. Lemma 8.1 also has a more subtle converse. If the linear functional $v$ in $B$ has the property that $(8.1)$ holds for every sequence $\left(w_{n}\right)$ that converges to zero in the weak* topology of $B$ and satisfies $\lim \sup _{n \rightarrow \infty}\left\|w_{n}\right\| \leq\|v\|$, then $v \in B_{0}$. Thus Lemma 8.1 characterizes the subspace $B_{0}$. We shall prove this in a forthcoming paper.

\section{WEAK* CONTINUITY OF ISOMETRIES AND PROOF OF THE ADJOINTNESS THEOREM}

We begin with

Lemma 9.1. Let $X$ and $Y$ be Riemann surfaces. If $T$ is an invertible $\mathbb{C}$-linear isometry of $Q^{*}(X)$ onto $Q^{*}(Y)$, then $T$ is sequentially continuous with respect to the weak* topologies.

Proof. Let $\left(w_{n}\right)$ be a sequence in $Q^{*}(X)$ that converges to 0 in the weak* topology. We must show that the sequence $\left(T w_{n}\right)$ converges (weak*) to 0 in $Q^{*}(Y)$. By the uniform boundedness principle there is a positive number $C$ so that $\left\|w_{n}\right\| \leq C$ for all $n$. Choose $v$ in $Q^{*}(X)_{0}$ so that $\|v\|>C$. Since $v$ and $\left(w_{n}\right)$ satisfy the hypotheses of Lemma 8.1, formula (8.1) holds. In addition, since $v \in W$, Lemmas 7.1 and 7.3 imply that $v$ is an extreme point of the closed ball in $Q^{*}(X)$ with center 0 and radius $\|v\|$. Since $T$ is an isometry, $T v$ is an extreme point of the closed ball in $Q^{*}(Y)$ with center 0 and radius $\|T v\|$, and

$$
\left\|T v \pm T w_{n}\right\| \rightarrow\|T v\|(=\|v\|) \text { as } n \rightarrow \infty
$$

Lemma 8.2 therefore implies that $\left(T w_{n}\right)$ converges (weak*) to 0 .

Proof of the adjointness theorem. Let an invertible $\mathbb{C}$-linear isometry $T: Q^{*}(X) \rightarrow$ $Q^{*}(Y)$ be given. We must find a $\mathbb{C}$-linear map $S: Q(Y) \rightarrow Q(X)$ such that $T=S^{*}$. The adjoint $\left(T \mid Q^{*}(X)_{0}\right)^{*}$ of the restriction of $T$ to $Q^{*}(X)_{0}$ is a $\mathbb{C}$-linear map of $Q^{*}(Y)^{*}=Q(Y)^{* *}$ into $\left(Q^{*}(X)_{0}\right)^{*}(=Q(X)$, by Lemma 6.3). The restriction of that adjoint to $Q(Y) \subset Q(Y)^{* *}$ is a $\mathbb{C}$-linear map $S: Q(Y) \rightarrow Q(X)$ satisfying

$$
v(S \varphi)=(T v)(\varphi)
$$


for $\varphi \in Q(Y)$ and $v \in Q^{*}(X)_{0}$. We claim that $T=S^{*}$. To verify the claim we must show that (9.1) holds for every $\varphi \in Q(Y)$ and $v \in Q^{*}(X)$. Let $\varphi$ and $v$ be given. By Lemma 6.2 there is a sequence $\left(v_{n}\right)$ in $Q^{*}(X)_{0}$ such that $v_{n} \rightarrow v$ (weak*). Lemma 9.1 implies that $T v_{n} \rightarrow T v\left(\right.$ weak $\left.^{*}\right)$. Therefore

$$
v(S \varphi)=\lim _{n \rightarrow \infty} v_{n}(S \varphi)=\lim _{n \rightarrow \infty}\left(T v_{n}\right)(\varphi)=(T v)(\varphi)
$$

\section{TEICHMÜLLER DISKS}

Our proof of the uniqueness theorem depends on some facts about Teichmüller disks. Let $X$ be any Riemann surface. A Beltrami form $\mu$ in $M(X)$ is called a Teichmüller differential if it equals $t|\varphi| / \varphi$, where $\varphi$ in $Q(X)$ is not identically equal to zero and the complex number $t$ belongs to the open unit disk $\Delta$. (Unlike many authors we allow $t=0$.) A Teichmüller differential is uniquely extremal. In other words, if $\mu$ and $\nu$ in $M(X)$ determine the same point in Teich $(X)$, and if $\mu$ is a Teichmüller differential, then $\|\nu\|_{\infty}>\|\mu\|_{\infty}$ unless $\nu=\mu$ (see Theorem 3 of Chapter 6 in [G2]). A quasiconformal homeomorphism $f$ of $X$ onto a Riemann surface $Y$ is called a Teichmüller map if its Beltrami coefficient $\mu_{f}$ is a Teichmüller differential.

Definitions. Let $X$ and $Y$ be Riemann surfaces. If $\varphi$ in $Q(X)$ is not identically zero, the Teichmüller disk $D_{\varphi}$ is the set of points in Teich $(X)$ determined by the Teichmüller differentials $t|\varphi| / \varphi$ with $t$ in $\Delta$. A Teichmüller disk in Teich $(Y)$ is a set $D$ of the form $\rho_{f}\left(D_{\varphi}\right)$ for some geometric isomorphism $\rho_{f}$ :Teich $(X) \rightarrow$ Teich $(Y)$, where $f$ is a quasiconformal map of $X$ onto $Y$.

We shall use the notion of the pull-back of a quadratic differential $\varphi$ in $Q(X)$ by a quasiconformal homeomorphism $f: Y \rightarrow X$. By definition $f^{*}(\varphi)$ is the integrable (but not necessarily holomorphic) quadratic differential on $Y$ such that

$$
f^{*}(\varphi)=(g \circ f)(\partial(h \circ f))^{2} \quad \text { in } f^{-1}(U)
$$

whenever $U$ is an open subset of $X, g$ and $h$ are holomorphic functions on $U$, and $\varphi=g(d h)^{2}$ in $U$. This equals the usual pull-back if $f$ is holomorphic.

Lemma 10.1 lists some familiar properties of Teichmüller disks. We include their proofs because we could not find a convenient reference.

Lemma 10.1. Let $g:$ Teich $(X) \rightarrow$ Teich $(Y)$ be a geometric isomorphism, and let $D_{\varphi}$ be a Teichmüller disk in Teich $(X)$. If $g\left(D_{\varphi}\right)$ contains the basepoint of Teich $(Y)$, then there are a Teichmüller map $f: Y \rightarrow X$ and an $s$ in $\Delta$ with the following properties:

$$
\psi=f^{*}(\varphi) \text { belongs to } Q(Y),
$$

$$
\mu_{f}=-s|\psi| / \psi,
$$




$$
g\left(\Phi_{X}(t|\varphi| / \varphi)\right)=\Phi_{Y}\left(\left(\frac{t-s}{1-\bar{s} t}\right)|\psi| / \psi\right) \quad \text { for all } \quad t \in \Delta .
$$

In particular, $g\left(D_{\varphi}\right)=D_{\psi}$.

Proof. If the geometric isomorphism $g$ preserves basepoints, there is a holomorphic isomorphism $f$ of $Y$ onto $X$ such that $g^{-1}=\rho_{f}$. Formula (4.2) in Section 4 (with the roles of $X$ and $Y$ reversed) shows that $\Phi_{Y}\left(f^{*}(\mu)\right)=g\left(\Phi_{X}(\mu)\right)$ for all $\mu$ in $M(X)$. Therefore (10.3) and (10.4) hold with $s=0$ and $\psi=f^{*}(\varphi)$.

If $g$ does not preserve basepoints, we choose $s \neq 0$ in $\Delta$ so that $g$ maps $\Phi_{X}(s|\varphi| / \varphi)$ to the basepoint of Teich $(Y)$. Then $g=\rho_{h}$, where $h: X \rightarrow Y$ is a Teichmüller map whose Beltrami coefficient is $s|\varphi| / \varphi$.

Set $f=h^{-1}$, so that $g^{-1}=\rho_{f}$. The local representation of the Teichmüller map $h$ (see for instance Theorem 8.1 in [Le]) shows that the quadratic differential $\psi=$ $f^{*}(\varphi)$ is holomorphic and that $f$ is a Teichmüller map whose Beltrami coefficient is $-s|\psi| / \psi$. This proves (10.1), (10.2), and (10.3).

Now $g\left(\Phi_{X}(t|\varphi| / \varphi)\right)$ is the equivalence class of $f_{t} \circ f$, where the Beltrami coefficient of $f_{t}$ is $t|\varphi| / \varphi$. A chain rule calculation using (10.2) and (10.3) shows that the Beltrami coefficient of $f_{t} \circ f$ is

$$
\mu_{t}=\left(\frac{t-s}{1-\bar{s} t}\right)(|\psi| / \psi)
$$

This proves (10.4).

Corollary 1. If the Teichmüller disk $D$ in Teich $(Y)$ contains the basepoint, then $D=D_{\psi}$ for some $\psi$ in $Q(Y)$.

Corollary 2. Let $D_{\varphi}$ be a Teichmüller disk in Teich $(X)$ and let $Q C(\varphi)$ be the set of Teichmüller maps $f$ of $X$ onto itself such that $f^{*}(\varphi)$ is a scalar multiple of $\varphi$ and $\mu_{f}$ is a scalar multiple of $|\varphi| / \varphi$. Then

(a) $Q C(\varphi)$ is a group under composition,

(b) the geometric automorphisms of Teich $(X)$ that map $D_{\varphi}$ onto itself are precisely the automorphisms $\rho_{f}, f$ in $Q C(\varphi)$, and

(c) if $f \in Q C(\varphi), f^{*}(\varphi)=a^{2} \varphi$, and $\mu_{f}=\frac{b}{a} \frac{|\varphi|}{\varphi}$, then

$$
\rho_{f}(\Phi(t|\varphi| / \varphi))=\Phi\left(\left(\frac{a t-b}{-\bar{b} t+\bar{a}}\right)|\varphi| / \varphi\right) \quad \text { for all } \quad t \in \Delta \text {. }
$$

Proof. Chain rule calculations similar to the verification of (10.4) above show that $Q C(\varphi)$ is a group and that $\rho_{f}$ maps $D_{\varphi}$ onto itself if $f \in Q C(\varphi)$.

Conversely, suppose the geometric automorphism $g$ maps $D_{\varphi}$ onto itself. Lemma 10.1 gives us a Teichmüller map $f$ of $X$ onto itself and a point $s$ in $\Delta$ such that $g=$ $\rho_{f}, \psi=f^{*}(\varphi)$ belongs to $Q(X), \mu_{f}=-s|\psi| / \psi$, and $g\left(D_{\varphi}\right)=D_{\psi}$. The Teichmüller disks $D_{\psi}$ and $D_{\varphi}$ therefore coincide. Since Teichmüller differentials are uniquely extremal, there is a complex number $\alpha$ such that $|\alpha|=1$ and $|\psi| / \psi=\alpha|\varphi| / \varphi$. Therefore $\psi$ is a scalar multiple of $\varphi$, and $f \in Q C(\varphi)$.

Finally, under the assumptions in (c) formula (10.5) reduces to (10.4).

Lemma 10.2 and its corollaries describe the behavior of Teichmüller disks under the action of an automorphism $F$ that satisfies the hypotheses of the uniqueness theorem. 
Lemma 10.2. Let $F$ be a basepoint-preserving holomorphic automorphism of Teich $(X)$ such that $F^{\prime}([i d])=\alpha I$ for some $\alpha$ in $\mathbb{C}$. If $D=D_{\varphi}$ is a Teichmüller disk in Teich $(X)$ containing the basepoint, then $F(D)=D$ and

$$
F(\Phi(t|\varphi| / \varphi))=\Phi(\alpha t|\varphi| / \varphi) \text { for all } t \in \Delta
$$

Proof. The holomorphic map $f: \Delta \rightarrow$ Teich $(X)$ defined by $f(t)=F(\Phi(t|\varphi| / \varphi)), t$ in $\Delta$, satisfies $f(0)=[i d]$ and

$$
f^{\prime}(0)=F^{\prime}([i d])\left(\Phi^{\prime}(0)(|\varphi| / \varphi)\right)=\alpha\left(\Phi^{\prime}(0)(|\varphi| / \varphi)\right) .
$$

By Theorem 2 of $[\mathrm{EKK}]$, there is a holomorphic map $\tilde{f}: \Delta \rightarrow M(X)$ such that $\widetilde{f}(0)=0$ and $f=\Phi \circ \tilde{f}$. Therefore

$$
\Phi^{\prime}(0)\left(\widetilde{f^{\prime}}(0)\right)=f^{\prime}(0)=\Phi^{\prime}(0)(\alpha|\varphi| / \varphi),
$$

and $\left(\widetilde{f^{\prime}}(0), \psi\right)_{X}=(\alpha|\varphi| / \varphi, \psi)_{X}$ for all $\psi$ in $Q(X)$. Taking $\psi=\bar{\alpha} \varphi$, and noting that $|\alpha|=1$, we obtain

$$
\iint_{X}\left(|\varphi|-\bar{\alpha} \widetilde{f}^{\prime}(0) \varphi\right)=0 .
$$

Since $\left\|\tilde{f}^{\prime}(0)\right\|_{\infty} \leq 1$ by Schwarz's lemma, (10.7) implies that $\tilde{f}^{\prime}(0)=\alpha|\varphi| / \varphi$. Since $\alpha|\varphi| / \varphi$ is an extreme point of the closed unit ball in $\operatorname{Belt}(X)$, the strong form of Schwarz's lemma (see [D, page 95]) implies that $\widetilde{f}(t)=\alpha t|\varphi| / \varphi$ for all $t$ in $\Delta$. That proves formula (10.6), which in turn implies that $F(D)=D$.

Corollary 1. Let $F$ be a holomorphic isomorphism of Teich $(X)$ onto Teich $(Y)$. Let $\tau$ be a point in Teich $(X)$. Suppose $g:$ Teich $(X) \rightarrow$ Teich $(Y)$ is a geometric isomorphism such that $F(\tau)=g(\tau)$ and $F^{\prime}(\tau)=\alpha g^{\prime}(\tau)$ for some $\alpha$ in $\mathbb{C}$. If $D$ is a Teichmüller disk in Teich $(X)$ and $\tau \in D$, then $F(D)$ is the Teichmüller disk $g(D)$.

Proof. By composing $F$ with an appropriate geometric isomorphism we may assume that $\tau$ is the basepoint of Teich $(X)$. The map $h=g^{-1} \circ F$ is a holomorphic automorphism of Teich $(X)$ such that $h(\tau)=\tau$ and $h^{\prime}(\tau)=\alpha I$. Let $D$ be a Teichmüller disk in Teich $(X)$ that contains $\tau$. Since $\tau$ is the basepoint, Corollary 1 to Lemma 10.1 says that $D=D_{\varphi}$ for some nonzero $\varphi$ in $Q(X)$. Therefore $h(D)=D$, by Lemma 10.2.

Corollary 2. Let $X$ be a Riemann surface with the isometry property. Suppose $F$ is a basepoint-preserving holomorphic automorphism of Teich $(X)$ such that $F^{\prime}([i d])=$ $\alpha I$ for some $\alpha$ in $\mathbb{C}$. Let $D=D_{\varphi}$ be a Teichmüller disk in Teich $(X)$ containing the basepoint. Then for each fixed $t$ in $\Delta$ there is a geometric automorphism $g_{t}$ of $\operatorname{Teich}(X)$ such that

$$
g_{t}\left(D_{\varphi}\right)=D_{\varphi} \text { and } g_{t}(\Phi(t|\varphi| / \varphi))=\Phi(\alpha t|\varphi| / \varphi) .
$$

Proof. Lemma 10.2 tells us that $F$ maps $D$ onto itself and satisfies (10.6). Fix any $t$ in $\Delta$. Applying Lemma 5.1 to $F$ with $\tau=\Phi(t|\varphi| / \varphi)$, we obtain a geometric automorphism $g_{t}$ of Teich $(X)$ such that $F(\tau)=g_{t}(\tau)$ and $F^{\prime}(\tau)=\alpha_{t} g_{t}{ }^{\prime}(\tau)$ for some $\alpha_{t}$ in $\mathbb{C}$. By Corollary $1, g_{t}(D)=F(D)=D$. 


\section{Special Teichmüller Disks AND \\ THE PROOF OF THE UNIQUENESS THEOREM}

Corollary 2 of Lemma 10.2 states a property of Teichmüller disks that we shall formalize in the following

Definition. Let $\alpha$ be a complex number of absolute value one. We say that the Teichmüller disk $D_{\varphi}$ in Teich $(X)$ has the $\alpha$ t-property if for each $t$ in $\Delta$ there is a geometric automorphism $g_{t}$ of $\operatorname{Teich}(X)$ such that $g_{t}\left(D_{\varphi}\right)=D_{\varphi}$ and $g_{t}(\Phi(t|\varphi| / \varphi))=$ $\Phi(\alpha t|\varphi| / \varphi)$. Notice that $g_{t}$ is allowed to vary with $t$.

Recall that a Riemann surface $X$ is said to have finite analytic type if it is isomorphic to $W \backslash E$, where $W$ is a compact Riemann surface and $E$ is a finite subset of $W$. If the genus of $W$ is $g$ and the number of points in $E$ is $n$, we say that $X$ has type $(g, n)$. Our final main result is

Theorem 7 (the disk theorem). Let $X$ be any Riemann surface of infinite analytic type. There is a Teichmüller disk $D$ through the basepoint of Teich $(X)$ that has the $\alpha$-property only for $\alpha=1$.

We shall prove the disk theorem in Sections 12 through 15. In this section we shall use it to prove the uniqueness theorem. Let $X$ be a Riemann surface with the isometry property, and let $F$ be a basepoint-preserving automorphism of Teich $(X)$ such that $F^{\prime}([i d])=\alpha I$ for some $\alpha$ in $\mathbb{C}$. We must show that $F$ is the identity.

This result is well known if $X$ has finite analytic type $(g, n)$. Here is a sketch of the proof. Since $X$ has the isometry property, types $(0,4),(1,1)$, and $(1,2)$ are excluded, and for type $(0,3)$ there is nothing to prove. For all other types $(g, n)$ we know by Royden's theorem (see Chapter 9 of [G2]) that $F=\rho_{f}$ for some holomorphic automorphism $f$ of $X$, and Lemma 4.2 implies that $f^{*}(\varphi)=\alpha \varphi$ for all $\varphi$ in $Q(X)$. For each $x$ in $X$ there is $\varphi_{x}$ in $Q(X)$ with a zero at $x$ of order at least $3 g+n-4$. If $2 g+n>4$, the equation $f^{*}\left(\varphi_{x}\right)=\alpha \varphi_{x}$ implies that $f$ fixes $x$, so $f$ is the identity and so is $F$. The only remaining type is $(2,0)$. In that case $X$ has a hyperelliptic involution $h$. If $x$ is not a fixed point of $h$, then $\varphi_{x}$ has double zeros at $x$ and $h(x)$ and no other zeros, so $f$ is either the identity or $h$. In either case $F$ is the identity, as required.

Now suppose $X$ has infinite analytic type. Choose a Teichmüller disk $D=D_{\varphi}$ through $[i d]$ satisfying the conclusion of the disk theorem. Corollary 2 of Lemma 10.2 tells us that $D$ has the $\alpha t$-property. (We know $|\alpha|=1$ because $F^{\prime}([i d])$ is an isometry.) Our choice of $D$ implies that $\alpha=1$ and $F^{\prime}([i d])$ is the identity.

The Bers embedding (see [G2] or [Na]) shows that Teich $(X)$ is holomorphically equivalent to a bounded region in a complex Banach space. Therefore, by the Cartan uniqueness theorem ([H, section 8$]$ ), any holomorphic automorphism of Teich $(X)$ is uniquely determined by its one-jet at a single point. We conclude that $F$ is the identity map. This proves the uniqueness theorem, given the disk theorem.

\section{Proof of the Disk THEOREM: CASE ONE}

In this section $X$ is the unit disk or the punctured unit disk $\Delta \backslash\{0\}$.

Let $h$ be a conformal isomorphism of $\Delta$ onto the bounded region

$$
U=\Delta \cap\{z \in \mathbb{C} ; \operatorname{Re}(z)<1 / 2\}
$$


Obviously $\varphi=(d h)^{2}$ belongs to $Q(X)$. We claim that the Teichmüller disk $D_{\varphi}$ does not have the $\alpha t$-property unless $\alpha=1$. Our proof will use the group $Q C(\varphi)$ defined in Corollary 2 to Lemma 10.1. We also need the group $A f f^{+}(\mathbb{C})$ of orientationpreserving affine transformations

$$
z \mapsto a z+b \bar{z}+c, \quad a, b, c \in \mathbb{C} \text { and }|a|>|b|,
$$

of $\mathbb{C}$ onto itself.

Lemma 12.1. For each $f$ in $Q C(\varphi)$ the homeomorphism $A_{f}=h \circ f \circ h^{-1}$ maps $U$ onto itself and belongs to $\mathrm{Aff}^{+}(\mathbb{C})$.

Proof. Clearly $A_{f}$ is an orientation-preserving homeomorphism of $h(X)$ onto itself. We must prove that it is the restriction to $h(X)$ of an affine transformation.

Let $f$ belong to $Q C(\varphi)$. Then $\mu_{f}=s \overline{d h} / d h$ for some $s$ in $\Delta$. In addition, since $f^{*}(\varphi)$ is a scalar multiple of $\varphi$, there is a nonzero complex number $a$ such that

$$
\partial(h \circ f)=a d h
$$

Put $H=a h+s a \bar{h}$. Then $\bar{\partial} H / \partial H=s \overline{d h} / d h=\mu_{f}$, so $H=h^{\sharp} \circ f$, for some holomorphic function $h^{\sharp}$ on $\Delta$.

Now $\partial\left(h^{\sharp} \circ f\right)=\partial H=a d h=\partial(h \circ f)$, by (12.1), so $d h^{\sharp}=d h$ (since $\partial f \neq 0$ a.e.). Therefore $h=h^{\sharp}+c$ for some $c$ in $\mathbb{C}$, and

$$
A_{f} \circ h=h \circ f=H+c=a h+s a \bar{h}+c .
$$

Lemma 12.2. If $A$ in $A f f^{+}(\mathbb{C})$ maps $U$ onto itself, then $A$ is the identity map.

Proof. Let $\sigma=\exp (\pi i / 3)$. Since $A$ is smooth and maps the boundary of $U$ onto itself, it fixes the two-point set $\{\sigma, \bar{\sigma}\}$. Orientation considerations show that it fixes the vertical line through $\sigma$ and $\bar{\sigma}$ pointwise. The tangent line to $S^{1}$ at the point $A(-1)$ is vertical, so $A(-1)=-1$ and $A$ fixes the real axis pointwise. Therefore $A$ is the identity.

Now suppose $f$ belongs to $Q C(\varphi)$. Lemmas 12.1 and 12.2 imply that $h \circ f \circ h^{-1}$ is the identity map. Therefore $f$ is the identity map, and so is $\rho_{f}$. By Corollary 2 to Lemma 10.1, every geometric automorphism of Teich $(X)$ that maps $D_{\varphi}$ onto itself equals $\rho_{f}$ for some $f$ in $Q C(\varphi)$. Therefore $D_{\varphi}$ does not have the $\alpha$-property unless $\alpha=1$.

This proves the disk theorem for $X=\Delta$ or $\Delta \backslash\{0\}$. The same proof works if $X=\Delta \backslash K$ and $K$ is any compact subset of $\Delta$, but for the purpose of generalization we prefer a proof that does not require the existence of a function like $h$.

\section{Proof OF THE DISK THEOREM: CASE TWO}

In this section $X$ will be the annulus

$$
A_{R}=\{\zeta \in \mathbb{C} ; 1<|\zeta|<R\}, \quad 1<R<\infty .
$$

As we shall see in Section 15, the proof given here is also useful in the general case. 
Lemma 13.1. Consider the quadratic differential $\varphi=-(d \zeta / \zeta)^{2}$ on the annulus $A_{R}$. Let $\tau_{1}=\Phi\left(t_{1}|\varphi| / \varphi\right)$ and $\tau_{2}=\Phi\left(t_{2}|\varphi| / \varphi\right)$ be points in the Teichmüller disk $D_{\varphi}$. If $g$ is a geometric automorphism of Teich $\left(A_{R}\right)$ such that $g\left(D_{\varphi}\right)=D_{\varphi}$ and $g\left(\tau_{1}\right)=\tau_{2}$, then

$$
t_{2}=\left(\frac{1-\bar{t}}{1-t}\right)\left(\frac{t_{1}+t}{\bar{t} t_{1}+1}\right)
$$

for some $t$ in $\Delta$ such that $|1-t|^{2}=1-|t|^{2}$.

Proof. We may assume $g=\rho_{f}$ for some $f$ in $Q C(\varphi)$. For each $t$ in $\Delta$ let $f_{t}: A_{R} \rightarrow \mathbb{C}$ be the quasiconformal homeomorphism

$$
f_{t}(\zeta)=|\zeta|^{2 t /(1-t)} \zeta=\zeta \exp \left(2 t(1-t)^{-1} \log |\zeta|\right), \quad \zeta \in A_{R}
$$

Then $\mu_{f_{t}}=-t|\varphi| / \varphi$ and $f_{t}\left(A_{R}\right)$ is the annulus $A_{R(t)}$, where

$$
R(t)=R^{\left(1-|t|^{2}\right) /|1-t|^{2}} .
$$

Any $f$ in $Q C(\varphi)$ has the form $f=h \circ f_{t}$, where $h: A_{R(t)} \rightarrow A_{R}$ is an isomorphism. We must therefore have $R(t)=R$, so $|1-t|^{2}=1-|t|^{2}$ and $h$ is an automorphism of $A_{R}$. Therefore $f^{*}(\varphi)=f_{t}^{*}\left(h^{*}(\varphi)\right)=f_{t}^{*}(\varphi)=(1-t)^{-2} \varphi$. Since $\mu_{f}=-t|\varphi| / \varphi$, (13.1) follows from formula (10.5).

Corollary. Let $A_{R}$ and $\varphi$ be as above. The Teichmüller disk $D_{\varphi}$ does not have the $\alpha$-property unless $\alpha=1$.

Proof. For any $t$ in $\Delta$ the Möbius transformation

$$
T_{t}(z)=\left(\frac{1-\bar{t}}{1-t}\right)\left(\frac{z+t}{\bar{t} z+1}\right)
$$

maps $\Delta$ onto itself and fixes the point -1 . If $|1-t|^{2}=1-|t|^{2}$, then $T_{t}{ }^{\prime}(-1)=1$, so $T_{t}$ is parabolic and maps every horocycle through -1 onto itself. Therefore the points $t_{1}$ and $t_{2}$ in (13.1) lie on the same horocycle through -1 . If $t_{1}$ is on the negative real axis, then $\left|t_{2}\right|>\left|t_{1}\right|$ unless $t=0$ and $t_{2}=t_{1}$. Therefore $D_{\varphi}$ does not have the $\alpha$-property unless $\alpha=1$.

This proves the disk theorem for the annulus $A_{R}$.

\section{Jenkins-Strebel DIFFERENTIALS}

The proof of the disk theorem in the general case is modelled on the proof for the annulus. Our tool for reducing the general case to the annulus is the theory of Jenkins-Strebel differentials. We shall need to consider only Jenkins-Strebel differentials whose regular closed trajectories sweep out one cylinder. We normalize them so that every regular closed trajectory has length $2 \pi$.

Definition. Let $\ell$ be a simple closed curve on the Riemann surface $X$. We call $\varphi$ in $Q(X)$ a Jenkins-Strebel differential for $\ell$ if there exist an annulus $A_{R}$ and an injective holomorphic map $h: A_{R} \rightarrow X$ such that $h^{*}(\varphi)=-(d \zeta / \zeta)^{2}$, the set $X \backslash h\left(A_{R}\right)$ has measure zero, and the loop $h\left(\left\{\zeta \in \mathbb{C} ;|\zeta|^{2}=R\right\}\right)$, properly oriented, is freely homotopic to $\ell$.

Note that we require Jenkins-Strebel differentials to have finite norm. The general theory in $\S 21$ of [S3] implies the existence of a Jenkins-Strebel differential for any simple closed Poincaré geodesic on $X$. Unless it is isomorphic to $\Delta$ or $\Delta \backslash\{0\}$, a Riemann surface of infinite analytic type has such a geodesic. To complete the proof of the disk theorem, it therefore suffices to prove the following lemma. 
Lemma 14.1. Let $\varphi$ be a Jenkins-Strebel differential for the simple closed curve $\ell$ on the Riemann surface $X$. If $X$ has infinite analytic type, then the Teichmüller disk $D_{\varphi}$ has the $\alpha$-property only for $\alpha=1$.

As a first step toward proving Lemma 14.1 we shall prove

Lemma 14.2. Let $\varphi$ be a Jenkins-Strebel differential for the simple closed curve $\ell$ on the Riemann surface $X$. Let a be a complex number with nonzero imaginary part. If $a^{2} \varphi$ is a Jenkins-Strebel differential for some simple closed curve on $X$, then $X$ has finite analytic type.

Proof. Let $h: A_{R} \rightarrow X$ be a holomorphic injection such that $h^{*}(\varphi)=-(d \zeta / \zeta)^{2}$ and the set $X \backslash h\left(A_{R}\right)$ has measure zero. Choose a horizontal strip $S$ in $\mathbb{C}$ so that $z \mapsto \zeta=\exp (2 \pi i z)$ maps $S$ onto $A_{R}$. Then $g(z)=h(\exp (2 \pi i z))$ maps $S$ onto $h\left(A_{R}\right)$, satisfies $g^{*}(\varphi)=4 \pi^{2}(d z)^{2}$, and has the property that $g(z)=g\left(z^{\prime}\right)$ if and only if $z-z^{\prime}$ is an integer.

If $a^{2} \varphi$ is also a Jenkins-Strebel differential for some simple loop on $X$, there exist a horizontal strip $S_{a}$ and a holomorphic map $g_{a}: S_{a} \rightarrow X$ with similar properties: the set $X \backslash g_{a}\left(S_{a}\right)$ has measure zero, $g_{a}(w)=g_{a}\left(w^{\prime}\right)$ if and only if $w-w^{\prime}$ is an integer, and $g_{a}{ }^{*}\left(a^{2} \varphi\right)=4 \pi^{2}(d w)^{2}$.

If $\varepsilon$ and $b$ are constants such that $\varepsilon= \pm 1$, we define $f_{\varepsilon, b}: \mathbb{C} \rightarrow \mathbb{C}$ by $f_{\varepsilon, b}(z)=$ $\varepsilon a z+b$. Almost every point in $S$ has a connected neighborhood $U$ on which there is a holomorphic map $f: U \rightarrow S_{a}$ with $g=g_{a} \circ f$ in $U$. Since $f^{*}\left((d w)^{2}\right)=a^{2}(d z)^{2}$, we must have $f=f_{\varepsilon, b}$ in $U$ for some $\varepsilon= \pm 1$ and $b$. Therefore

$$
g=g_{a} \circ f_{\varepsilon, b}
$$

in $U$, and $U$ is a subset of

$$
V=V(\varepsilon, b)=\left\{z \in S ; f_{\varepsilon, b}(z) \in S_{a}\right\} .
$$

Since $a$ is not real, $V$ is (the interior of) a parallelogram with an edge on each boundary line of $S$. These horizontal edges, except for their endpoints, are mapped into $S_{a}$ by $f_{\varepsilon, b}$ and correspond to points of $X$. The other two edges of $V$ lie on level lines of $z \mapsto \operatorname{Im}(a z)$. By analytic continuation, (14.1) holds everywhere in $V(\varepsilon, b)$.

If $z+1$ belongs to $V(\varepsilon, b)$, then

$$
g(z)=g(z+1)=g_{a}\left(f_{\varepsilon, b}(z+1)\right)=g_{a}\left(f_{\varepsilon, \varepsilon a+b}(z)\right),
$$

and that equation holds throughout $V(\varepsilon, \varepsilon a+b)$, which is the image of $V(\varepsilon, b)$ under translation to the left by one.

We have seen that the collection of parallelograms $V(\varepsilon, b)$ in which (14.1) holds is invariant under translation by one and covers almost all of $S$. It is easy to verify that all these parallelograms are congruent. We claim that no two of them overlap. Suppose that $W=V(\varepsilon, b) \cap V\left(\varepsilon^{\prime}, b^{\prime}\right)$ is not empty and that $g=g_{a} \circ f_{\varepsilon, b}=g_{a} \circ f_{\varepsilon^{\prime}, b^{\prime}}$ in $W$. Then the function $f_{\varepsilon, b}-f_{\varepsilon^{\prime}, b^{\prime}}$ is integer valued on $W$, so $\varepsilon=\varepsilon^{\prime}, b-b^{\prime}$ is an integer, and $V(\varepsilon, b)=V\left(\varepsilon^{\prime}, b^{\prime}\right)$, as we claimed. Therefore the parallelograms $V(\varepsilon, b)$ in which (14.1) holds pave $S$. Similarly, their images $f_{\varepsilon, b}(V(\varepsilon, b))$, together with the translates of these images by the integers, pave $S_{a}$.

Because of the translation invariance there is a positive integer $n$ such that each $V(\varepsilon, b)$ has width $1 / n$. Choosing $n$ adjacent parallelograms and identifying pairwise the edges corresponding to the same points in $X$ we obtain a compact Riemann surface $\widehat{X}$. Either $X=\widehat{X}$ or else we obtain $X$ by puncturing $\widehat{X}$ at some vertices of the parallelograms. In either case, $X$ has finite analytic type. 
Corollary. Suppose $X$ has infinite analytic type. If both $\varphi$ and $a^{2} \varphi$ are JenkinsStrebel differentials for simple loops on $X$, then $a^{2}=1$.

Proof. Lemma 14.2 tells us that $a$ is real. Define $g: S \rightarrow X, g_{a}: S_{a} \rightarrow X, f_{\varepsilon, b}$, and $V(\varepsilon, b)$ as in the proof of Lemma 14.2. Again (14.1) holds in some $V(\varepsilon, b)$. This time, since $a$ is real, $V(\varepsilon, b)$ is a horizontal strip. If $z$ belongs to $V(\varepsilon, b),(14.1)$ gives

$$
g_{a}(\varepsilon a z+b)=g(z)=g(z+\varepsilon)=g_{a}(\varepsilon a(z+\varepsilon)+b)=g_{a}(\varepsilon a z+b+a) .
$$

Therefore $a$ is an integer. Similarly, $1 / a$ is an integer, since

$$
g(z+(1 / a))=g_{a}(\varepsilon a z+\varepsilon+b)=g_{a}(\varepsilon a z+b)=g(z) .
$$

\section{Proof OF Lemma 14.1}

Let $\varphi$ be the Jenkins-Strebel differential for some simple loop $\ell$ on $X$. We learned the following description of the Teichmüller disk $D_{\varphi}$ from Marden and Masur, who used it for surfaces of finite analytic type (see $[\mathrm{MM}])$. Choose an annulus $A_{R}$ and an injective holomorphic map $h: A_{R} \rightarrow X$ such that $h^{*}(\varphi)=-(d \zeta / \zeta)^{2}$ and the set $X \backslash h\left(A_{R}\right)$ has measure zero. The Riemann surface $X$ and the Jenkins-Strebel differential $\varphi$ can be reconstructed from $A_{R}$ by adjoining to $A_{R}$ the boundary arcs that correspond to horizontal trajectories of $\varphi$ and identifying with each other the points of these arcs that correspond to the same point on $X$. In fact the identification space $\widehat{X}$ carries a unique Riemann surface structure such that the inclusion of $A_{R}$ in $\widehat{X}$ is a holomorphic map and the quadratic differential $-(d \zeta / \zeta)^{2}$ on $A_{R}$ extends to a Jenkins-Strebel differential for the simple loop $\left\{\zeta \in \mathbb{C} ;|\zeta|^{2}=R\right\}$ on $\widehat{X}$. We may assume that $X=\widehat{X}$ and that the restriction of $\varphi$ to $A_{R}$ is $-(d \zeta / \zeta)^{2}$.

For each $t$ in $\Delta$ let $f_{t}: A_{R} \rightarrow \mathbb{C}$ be the quasiconformal homeomorphism defined by (13.2) and let $R(t)$ be defined by (13.3). We know that $f_{t}$ maps $A_{R}$ onto $A_{R(t)}$. In addition, $f_{t}$ maps the boundary circles of $A_{R}$ homeomorphically onto the boundary circles of $A_{R(t)}$, preserving the angular measures of the arcs on the boundary. We can therefore construct an identification space $\widehat{X}_{t}$ with a Riemann surface structure, a Jenkins-Strebel differential $\varphi_{t}$ on $\widehat{X}_{t}$, and a Teichmüller map $\hat{f}_{t}$ of $X$ onto $\widehat{X}_{t}$ such that the restriction of $\varphi_{t}$ to $A_{R(t)}$ is $-(d \zeta / \zeta)^{2}$ and the restriction of $\hat{f}_{t}$ to $A_{R}$ is $f_{t}$. Computation in $A_{R}$ shows that $\mu_{\hat{f}_{t}}=-t|\varphi| / \varphi$ and $\hat{f}_{t}^{*}\left(\varphi_{t}\right)=(1-t)^{-2} \varphi$.

As usual, to understand the geometric automorphisms of Teich $(X)$ that map $D_{\varphi}$ onto itself we must study the group $Q C(\varphi)$. Let $f$ belong to that group. Then $f(X)=X$ and $\mu_{f}=-t|\varphi| / \varphi$ for some $t$ in $\Delta$. Therefore $f=g \circ \hat{f}_{t}$, where $g$ is an isomorphism of $\widehat{X}_{t}$ onto $X$. In addition, there is a constant $c$ such that

$$
c \varphi=f^{*}(\varphi)=\hat{f}_{t}^{*}\left(g^{*}(\varphi)\right)
$$

Since $\hat{f}_{t}^{*}\left(c(1-t)^{2} \varphi_{t}\right)$ also equals $c \varphi$, we must have $g^{*}(\varphi)=c(1-t)^{2} \varphi_{t}$.

Now $\varphi_{t}$ and $g^{*}(\varphi)$ are Jenkins-Strebel differentials on $\widehat{X}_{t}$, for the respective simple loops $\left\{\zeta \in \mathbb{C} ;|\zeta|^{2}=R(t)\right\}$ and $g^{-1}\left(\left\{\zeta \in \mathbb{C} ;|\zeta|^{2}=R\right\}\right)$. Since $\widehat{X}_{t}$ is quasiconformally equivalent to $X$, it has infinite analytic type. Therefore, by the corollary 
of Lemma 14.2, $g^{*}(\varphi)=\varphi_{t}$ and $c=(1-t)^{-2}$. This formula for $c$ reduces (15.1) to $f^{*}(\varphi)=(1-t)^{-2} \varphi$. In addition, since $g^{*}$ is an isometry,

$$
\iint_{A_{R}}|\zeta|^{-2} d \xi d \eta=\|\varphi\|=\left\|\varphi_{t}\right\|=\iint_{A_{R(t)}}|\zeta|^{-2} d \xi d \eta
$$

so $R(t)=R$ and $|1-t|^{2}=1-|t|^{2}$. We have proved that for any $f$ in $Q C(\varphi)$ there is $t$ in $\Delta$ satisfying

$$
\mu_{f}=-t|\varphi| / \varphi, \quad f^{*}(\varphi)=(1-t)^{-2} \varphi, \quad \text { and } \quad|1-t|^{2}=1-|t|^{2} .
$$

Now let $\tau_{1}=\Phi\left(t_{1}|\varphi| / \varphi\right)$ and $\tau_{2}=\Phi\left(t_{2}|\varphi| / \varphi\right)$ be points in the Teichmüller disk $D_{\varphi}$. Suppose $f$ in $Q C(\varphi)$ satisfies $\rho_{f}\left(\tau_{1}\right)=\tau_{2}$. Choose $t$ in $\Delta$ so that (15.2) holds. As in the proof of Lemma 13.1, formula (10.5) implies that $t_{1}$ and $t_{2}$ satisfy (13.1). Since $|1-t|^{2}=1-|t|^{2}$, the proof of the corollary of Lemma 13.1 shows that $D_{\varphi}$ does not have the $\alpha t$-property unless $\alpha=1$.

As we observed in $\S 14$, the proof of Lemma 14.1 completes the proof of the disk theorem. The uniqueness and automorphism theorems follow.

\section{RiEMANN SURFACES OF FINITE TOPOLOGICAL TYPE}

It remains to prove the isometry, classification, and isomorphism theorems. The crucial step is to prove the isometry theorem. Our proof, like the proof of Theorem 2.1 in [Ko], follows the strategy of Section 7.5 in Rudin's book [Ru1]. In order to adapt that strategy to our situation we need some technical facts about Riemann surfaces of finite topological type, which we shall state and prove in this section.

First we recall (see [FK] or [G2]) that if $X$ has finite topological type there is a compact bordered Riemann surface $\overline{X^{*}}$ with interior $X^{*}$ and (possibly empty) border $B$ such that $X$ is conformally equivalent to $X^{*} \backslash E$ for some finite subset $E$ of $X^{*}$. We shall identify $X$ with $X^{*} \backslash E$. Then $X^{*}$ and $X$ have the same border $B=\overline{X^{*}} \backslash X^{*}$. The analytic type of $X$ is finite if and only if $B$ is empty. A Riemann surface has finite analytic type if and only if its space of integrable quadratic differentials has finite dimension. (See [Kr, pages 324-335] for a careful proof of this fact.) The hypotheses of the isometry theorem guarantee that the dimension of $Q(X)$ is infinite and equals the dimension of $Q(Y)$. Therefore $Y$, like $X$, has nonempty border.

Lemma 16.1. Let $U$ be a nonempty open set in the compact Riemann surface $W$, and let $D$ be any divisor on $W$ whose support lies in $W \backslash U$. There is a meromorphic function $f$ on $W$ such that $D$ minus the divisor of $f$ is supported in $U$.

Proof. This well known fact follows trivially from Exercise 21.1 at the end of $\S 21$ in $[\mathrm{F}]$.

Lemma 16.2. Let $Y$ be a Riemann surface with finite topological type and with nonempty border $B$. Let $\widehat{Y}$ be the double of $Y$. There is a subregion $W$ of $\widehat{Y}$ such that $Y \cup B \subset W$ and the restriction map carries $Q(W)$ onto a dense subspace of $Q(Y)$.

Proof. Let $\pi: \Delta \rightarrow Y$ be a holomorphic covering map. The group of covering transformations is a finitely generated Fuchsian group $\Gamma$ of the second kind. The 
limit set $L(\Gamma)$ is a closed nowhere dense subset of $S^{1}$, and $\pi$ extends to a holomorphic covering map $\widehat{\pi}: \widehat{\mathbb{C}} \backslash L(\Gamma) \rightarrow \widehat{Y}$. The border $B$ of $Y$ is the boundary of $Y$ in $\widehat{Y}$ and consists of $n \geq 1$ disjoint analytic simple closed curves. The inverse image of $B$ under $\widehat{\pi}$ is $S^{1} \backslash L(\Gamma)$.

Choose a subregion $W$ of $\widehat{Y}$ with the following four properties: (i) $Y \cup B \subset W$, (ii) the boundary $\partial W$ consists of $n$ disjoint analytic simple closed curves in $\widehat{Y}$, (iii) the set $(W \cup \partial W) \backslash Y$ consists of $n$ disjoint closed annuli, each of which is bounded by one component of $B$ and one component of $\partial W$, and (iv) $\Omega=\widehat{\pi}^{-1}(W)$ is a bounded simply connected region in $\mathbb{C}$. (It is possible to do this so that $\Omega$ is a Jordan region whose boundary is the union of $L(\Gamma)$ and a collection of circular arcs whose endpoints belong to $L(\Gamma)$. See $[\mathrm{EM}]$ and $[\mathrm{Kn}]$.)

The map $\widehat{\pi}: \Omega \rightarrow W$ induces an injective pull-back map $\widehat{\pi}^{*}$, which carries holomorphic quadratic differentials on $W$ to holomorphic quadratic differentials on $\Omega$. We denote the image $\widehat{\pi}^{*}(Q(W))$ by $Q(\Omega, \Gamma)$, and we norm $Q(\Omega, \Gamma)$ so that $\widehat{\pi}^{*}: Q(W) \rightarrow Q(\Omega, \Gamma)$ is an invertible isometry. Similarly we put $Q(\Delta, \Gamma)$ equal to $\pi^{*}(Q(Y))$, normed so that $\pi^{*}$ maps $Q(Y)$ isometrically onto $Q(\Delta, \Gamma)$. We want to prove that the restriction map carries $Q(\Omega, \Gamma)$ onto a dense subspace of $Q(\Delta, \Gamma)$.

According to Theorem 2 of $[\mathrm{B}]$, the Poincaré series operator

$$
\Theta(\varphi)=\sum_{\gamma \in \Gamma} \gamma^{*}(\varphi)
$$

defines bounded linear maps of $Q(\Omega)$ and $Q(\Delta)$ onto $Q(\Omega, \Gamma)$ and $Q(\Delta, \Gamma)$ respectively. The quadratic differentials $P(z) d z^{2}, P(z)$ a polynomial, are dense in $Q(\Delta)$ (see for example $[\mathrm{SW}]$ ) and belong to $Q(\Omega)$ because $\Omega$ is bounded. Therefore the quadratic differentials $\Theta\left(P(z) d z^{2}\right), P(z)$ a polynomial, belong to $Q(\Omega, \Gamma)$ and restrict to a dense subspace of $Q(\Delta, \Gamma)$.

Definition. Let $W$ be any Riemann surface. We denote by $H^{\infty}(W)$ the complex Banach algebra of bounded holomorphic functions on $W$, with the sup norm.

Lemma 16.3. Let $Y$ be as in Lemma 16.2. There is a quadratic differential $\varphi_{0}$ in $Q(Y)$ such that the set $\left\{f \varphi_{0} ; f \in H^{\infty}(Y)\right\}$ is dense in $Q(Y)$.

Proof. Let $\overline{Y^{*}}$ be a compact bordered Riemann surface with interior $Y^{*}$ such that $Y$ is the complement of a finite subset of $Y^{*}$. The double of $Y$ is therefore the complement of a finite subset of the double $\widehat{Y^{*}}$ of $Y^{*}$.

Choose a nontrivial meromorphic differential $\psi$ on $\widehat{Y^{*}}$. Applying Lemma 16.1 with $W=\widehat{Y^{*}}$ and $U$ the complement of $\overline{Y^{*}}$, choose a meromorphic function $f_{0}$ on $\widehat{Y^{*}}$ so that the meromorphic quadratic differential $f_{0} \psi$ has a simple pole at each point of $Y^{*} \backslash Y$ and no other zeros or poles in $\overline{Y^{*}}$. Let $\varphi_{0}$ be the restriction of $f_{0} \psi$ to $Y$. Clearly $f \varphi_{0}$ belongs to $Q(Y)$ for every $f$ in $H^{\infty}(Y)$. We claim that the set $\left\{f \varphi_{0} ; f \in H^{\infty}(Y)\right\}$ is dense in $Q(Y)$. In view of Lemma 16.2, it suffices to prove that $f=\varphi / \varphi_{0}$ belongs to $H^{\infty}(Y)$ whenever $\varphi$ is the restriction to $Y$ of some $\widetilde{\varphi}$ in $Q(W)$ and $W$ is a subregion of $\widehat{Y^{*}}$ that contains $Y \cup B$. That is easy. The integrability of $\varphi$ implies that $f$ has a removable singularity at each point of $Y^{*} \backslash Y$, and $\widetilde{\varphi} / \varphi_{0}$ provides a continuous extension of $f$ to the compact set $\overline{Y^{*}}$. 


\section{A lemma about bounded holomorphic FunCtions}

If $X$ and $Y$ are Riemann surfaces, every holomorphic map $h: X \rightarrow Y$ induces a multiplicative $\mathbb{C}$-linear map $h^{*}: H^{\infty}(Y) \rightarrow H^{\infty}(X)$ given by $h^{*}(f)=f \circ h$ if $f \in H^{\infty}(Y)$. We need a converse result, which extends Theorem 6.6.5 of [Ru1] to the setting of compact bordered Riemann surfaces. If $Y$ is the interior of a compact bordered Riemann surface $\bar{Y}$ with nonempty border, we denote by $A(Y)$ the closed subalgebra of $H^{\infty}(Y)$ consisting of the functions with continuous extensions to $\bar{Y}$.

Lemma 17.1. Let $X$ and $Y$ be the interiors of the compact bordered Riemann surfaces $\bar{X}$ and $\bar{Y}$. Let $L: H^{\infty}(Y) \rightarrow H^{\infty}(X)$ be a multiplicative complex linear map. Assume that there is at least one $f$ in $A(Y)$ such that the function $L(f)$ is not constant. Then there is a nonconstant holomorphic map $h: X \rightarrow Y$ such that $L(f)=h^{*}(f)$ for all $f$ in $H^{\infty}(Y)$.

Proof. We follow the proof of Theorem 6.6.5 in [Ru1] almost verbatim. Since $L$ is multiplicative and is not the zero map, $L(1)=1$. Therefore, if $p \in X$, the map $f \mapsto L(f)(p)$ is a homomorphism of $A(Y)$ onto $\mathbb{C}$. By Theorems 5.3 and 5.4 of $[\mathrm{A}]$, there is a unique point $h(p)$ in $\bar{Y}$ such that $L(f)(p)=f(h(p))$ for all $f$ in $A(Y)$.

Choosing $f$ so that $L(f)$ is not constant we see that the map $h: X \rightarrow \bar{Y}$ is not constant. Let $\widehat{Y}$ be the double of $Y$. We claim that $h$ is holomorphic, viewed as a map of $X$ into $\widehat{Y}$. To verify this claim, choose any $p$ in $X$. Using Lemma 16.1, choose a meromorphic function $f$ on $\widehat{Y}$ with a simple zero at $h(p)$ and no other zeros or poles in $\bar{Y}$. Then $f \in A(Y)$. In addition there exist a positive number $\epsilon$ and an open neighborhood $V$ of $h(p)$ in $\widehat{Y}$ such that $f$ maps $V$ homeomorphically onto $B(0, \epsilon)=\{z \in \mathbb{C} ;|z|<\epsilon\}$ and $|f| \geq \epsilon$ in $\bar{Y} \backslash V$. Now $f \circ h=L(f)$ in $X$, so $h$ coincides with the holomorphic map $(f \mid V)^{-1} \circ L(f)$ in the open neighborhood $L(f)^{-1}(B(0, \epsilon))$ of $p$. Therefore $h$ is holomorphic at $p$. That proves our claim. Since $h$ is a nonconstant holomorphic map its image is open in $\widehat{Y}$, so $h(X) \subset Y$.

Now choose any $g$ in $H^{\infty}(Y)$ and $p$ in $X$. Let $f$ be the function chosen in the previous paragraph and observe that the function $\widehat{g}=[g-g(h(p))] / f$ belongs to $H^{\infty}(Y)$. Now

$$
L(g)-g(h(p))=L(f \widehat{g})=L(f) L(\widehat{g})=(f \circ h) L(\widehat{g})
$$

Evaluation at $p$ shows that $L(g)(p)-g(h(p))=0$, so $L(g)=g \circ h=h^{*}(g)$.

\section{Proof of the isometry theOREM}

Our proof will proceed by adapting the reasoning in Section 7.5 of [Ru1] to the Riemann surface setting. Let $\overline{X^{*}}$ and $\overline{Y^{*}}$ be compact bordered Riemann surfaces with interiors $X^{*}$ and $Y^{*}$ such that $X$ and $Y$ are subsets of $X^{*}$ and $Y^{*}$ with finite complements.

Using Lemma 16.3 , choose $\varphi_{0}$ in $Q(Y)$ so that the set $\left\{f \varphi_{0} ; f \in H^{\infty}(Y)\right\}$ is dense in $Q(Y)$. Put $\psi_{0}=S\left(\varphi_{0}\right)$. Since $\varphi_{0}$ has positive norm, the norm of $\psi_{0}$ is also positive. Put

$$
L(f)=\frac{S\left(f \varphi_{0}\right)}{\psi_{0}} \quad \text { for all } f \in H^{\infty}\left(Y^{*}\right)
$$


Clearly $L(f)$ is well-defined as a meromorphic function on $X^{*}$, and the map $f \mapsto$ $L(f)$ is complex linear. Moreover, $L(1)=1$ and, since $S$ is an isometry,

$$
\iint_{X^{*}}|L(f)|\left|\psi_{0}\right|=\iint_{Y^{*}}|f|\left|\varphi_{0}\right|
$$

for all $f$ in $H^{\infty}\left(Y^{*}\right)$. Applying Theorem 7.5.3 in [Ru1] we conclude that the map $L$ is multiplicative and that $\|L(f)\|_{\infty}=\|f\|_{\infty}$ for all $f$ in $H^{\infty}\left(Y^{*}\right)$. The meromorphic function $L(f)$ therefore has no poles and belongs to $H^{\infty}\left(X^{*}\right)$.

If $L(f)$ is constant for every $f$ in $A\left(Y^{*}\right)$, then $S\left(f \varphi_{0}\right)$ is a constant multiple of $\psi_{0}$ for every $f$ in $A\left(Y^{*}\right)$. That is impossible because $S$ is injective and $A\left(Y^{*}\right)$ contains nonconstant functions (by Lemma 16.1). The map $L$ therefore satisfies the hypotheses of Lemma 17.1, so there is a nonconstant holomorphic map $h: X^{*} \rightarrow Y^{*}$ such that $L(f)=h^{*}(f)$ for all $f$ in $H^{\infty}\left(Y^{*}\right)$. Therefore

$$
S\left(f \varphi_{0}\right)=h^{*}(f) \psi_{0} \quad \text { for all } f \in H^{\infty}\left(Y^{*}\right) .
$$

Reversing the roles of $S$ and $S^{-1}$ and of $\varphi_{0}$ and $\psi_{0}$, and repeating the reasoning above, we obtain a nonconstant holomorphic map $\widetilde{h}: Y^{*} \rightarrow X^{*}$ such that

$$
S^{-1}\left(\tilde{f} \psi_{0}\right)=\widetilde{h}^{*}(\widetilde{f}) \varphi_{0} \quad \text { for all } \tilde{f} \in H^{\infty}\left(X^{*}\right)
$$

Applying $S$ to both sides of (18.2) and using (18.1), we find that

$$
\widetilde{f} \psi_{0}=S\left(\widetilde{h}^{*}(\widetilde{f}) \varphi_{0}\right)=h^{*}\left(\widetilde{h}^{*}(\widetilde{f})\right) \psi_{0}
$$

for all $\widetilde{f}$ in $H^{\infty}\left(X^{*}\right)$. Therefore

$$
\widetilde{f}(p)=h^{*}\left(\widetilde{h}^{*}(\widetilde{f})\right)(p)=\widetilde{f}(\widetilde{h}(h(p))) \quad \text { for all } \widetilde{f} \in H^{\infty}\left(X^{*}\right) \text { and } p \in X^{*} .
$$

Fixing $p$ and choosing $\tilde{f}$ to have a zero at $p$ and no other zeros in $X^{*}$ we conclude that $\widetilde{h} \circ h$ is the identity map on $X^{*}$. A similar argument, which begins by applying $S^{-1}$ to both sides of (18.1), shows that $h \circ \widetilde{h}$ is the identity on $Y^{*}$. Therefore $h$ and $\widetilde{h}$ are inverse holomorphic isomorphisms.

Put $T(\varphi)=\widetilde{h}^{*}(S(\varphi))$ for all $\varphi$ in $Q(Y)$. Then $T$ is a $\mathbb{C}$-linear isometry of $Q(Y)$ onto $Q\left(Y^{\prime}\right)$, where $Y^{\prime}=h(X) \subset Y^{*}$. If $f \in H^{\infty}\left(Y^{*}\right)$, (18.1) gives

$$
T\left(f \varphi_{0}\right)=\widetilde{h}^{*}\left(S\left(f \varphi_{0}\right)\right)=\widetilde{h}^{*}\left(h^{*}(f) \psi_{0}\right)=f \widetilde{h}^{*}\left(\psi_{0}\right)=f T\left(\varphi_{0}\right) .
$$

Since $T$ is an isometry,

$$
\iint_{Y^{*}}|f|\left|T\left(\varphi_{0}\right)\right|=\iint_{Y^{*}}|f|\left|\varphi_{0}\right|
$$

for all $f$ in $H^{\infty}\left(Y^{*}\right)$. Theorem 7.5.3 in [Ru1] therefore implies that

$$
\iint_{Y^{*}} f \bar{g}\left|T\left(\varphi_{0}\right)\right|=\iint_{Y^{*}} f \bar{g}\left|\varphi_{0}\right|
$$


for all $f$ and $g$ in $H^{\infty}\left(Y^{*}\right)$. The Stone-Weierstrass theorem implies that the linear space spanned by the functions $f \bar{g}, f$ and $g$ in $A\left(Y^{*}\right)$, is a dense subalgebra of the space of continuous functions on $\overline{Y^{*}}$. Therefore $\left|T\left(\varphi_{0}\right)\right|=\left|\varphi_{0}\right|$ in $Y^{*}$ and the meromorphic function $T\left(\varphi_{0}\right) / \varphi_{0}$ on $Y^{*}$ is a constant $\alpha$ of absolute value one.

We conclude from (18.3) that $T\left(f \varphi_{0}\right)=\alpha f \varphi_{0}$ for all $f$ in $H^{\infty}\left(Y^{*}\right)$. Since the set of these $f \varphi_{0}$ is dense in $Q(Y)$, it follows that $T$ equals $\alpha$ times the identity on $Q(Y)$. Therefore

$$
S(\varphi)=h^{*}(T(\varphi))=\alpha h^{*}(\varphi) \quad \text { for all } \varphi \in Q(Y)
$$

It remains only to prove that $h(X)=Y$ or, equivalently, that $h\left(X^{*} \backslash X\right)=Y^{*} \backslash Y$. This follows immediately from formula (18.4), for $X^{*} \backslash X$ is the set of points at which some $\psi$ in $Q(X)$ has a pole, and $Y^{*} \backslash Y$ is the set of points at which some $\varphi$ in $Q(Y)$ has a pole.

\section{Proof of the Classification THEOREM}

Let the Riemann surfaces $X$ and $Y$ have finite topological type, and suppose the border of $X$ is nonempty. Suppose there is a $\mathbb{C}$-linear isometry $L$ of the tangent space to Teich $(X)$ at $[f: X \rightarrow V]$ onto the tangent space to Teich $(Y)$ at $[g: Y \rightarrow W]$. Let $\rho_{f}: \operatorname{Teich}(X) \rightarrow$ Teich $(V)$ and $\rho_{g}: \operatorname{Teich}(Y) \rightarrow$ Teich $(W)$ be the geometric isomorphisms induced by $f$ and $g$. Then $\rho_{g}{ }^{\prime}[g] \circ L \circ \rho_{f}{ }^{\prime}[f]^{-1}$ is a $\mathbb{C}$-linear isometry of the tangent space to Teich $(V)$ at $[i d]$ onto the tangent space to Teich $(W)$ at $[i d]$. Identifying these tangent spaces with $Q^{*}(V)$ and $Q^{*}(W)$ as in Sections 3 and 4 , we obtain a $\mathbb{C}$-linear isometry $T$ of $Q^{*}(V)$ onto $Q^{*}(W)$. The adjointness theorem gives us a $\mathbb{C}$-linear isometry $S$ of $Q(W)$ onto $Q(V)$ such that $S^{*}=T$. Since $V$ and $W$ are quasiconformally equivalent to $X$ and $Y$, they have finite topological type and $V$ has nonempty border. Since $Q(W)$ and $Q(V)$ are isometric, the border of $W$ is also nonempty and the isometry theorem gives us a holomorphic isomorphism $h$ of $V$ onto $W$ and a complex number $\alpha$ such that $S(\varphi)=\alpha h^{*}(\varphi)$ for all $\varphi$ in $Q(W)$. The required quasiconformal homeomorphism of $X$ onto $Y$ is $g^{-1} \circ h \circ f$.

\section{Proof of the ISOMORPhism THEOREM}

Suppose the Riemann surfaces $X$ and $Y$ have finite topological type and the border of $X$ is nonempty. Let $F$ be a holomorphic isomorphism of Teich $(X)$ onto $\operatorname{Teich}(Y)$. Since $F^{\prime}([i d])$ is a $\mathbb{C}$-linear isometry from the tangent space to Teich $(X)$ at $[i d]$ onto the tangent space to Teich $(Y)$ at $F([i d])$, the classification theorem gives us a quasiconformal homeomorphism $f$ of $X$ onto $Y$. The isometry theorem implies that $X$ has the isometry property, so the automorphism theorem guarantees that the automorphism $F^{-1} \circ \rho_{f}$ of $\operatorname{Teich}(X)$ is geometric. Therefore $F$ is geometric. $\square$

\section{REFERENCES}

[A] R. Arens, The closed maximal ideals of algebras of functions holomorphic on a Riemann surface, Rend. Circ. Mat. Palermo (2)7 (1958), 245-260. MR 21:4242

[B] L. Bers, Automorphic forms and Poincaré series for infinitely generated Fuchsian groups, Amer. J. Math. 87 (1965), 196-214. MR 30:4937

[D] S. Dineen, The Schwarz Lemma, Oxford, 1989. MR 91f:46064

[E1] C. J. Earle, On holomorphic cross-sections in Teichmüller spaces, Duke Math. J. 36 (1969), 409-416. MR 40:7442 
[E2] The integrable automorphic forms as a dual space, Modular Functions in Analysis and Number Theory (T. Metzger, ed.), Lecture Notes in Mathematics and Statistics 5, University of Pittsburgh, 1983, pp. 30-40. MR 85i:30087

[E3] , The integrable automorphic forms as a dual space, II, Complex Variables Theory Appl. 12 (1989), 153-158. MR 91g:32045

[EK1] C. J. Earle and I. Kra, On holomorphic mappings between Teichmüller spaces, Contributions to Analysis (L. V. Ahlfors et al., eds.), Academic Press, New York, 1974, pp. 107-124. MR 55:3324

[EK2] _ On isometries between Teichmüller spaces, Duke Math. Journal 41 (1974), 583591. MR 50:596

[EKK] C. J. Earle, I. Kra, and S. L. Krushkal, Holomorphic motions and Teichmüller spaces, Trans. Amer. Math. Soc. 343 (1994), 927-948. MR 94h:32035

[EM] C. J. Earle and A. Marden, Projections to automorphic functions, Proc. Amer. Math. Soc. 19 (1968), 274-278. MR 37:412

[FK] H. M. Farkas and I. Kra, Riemann Surfaces, Second Edition, Springer-Verlag, New York, Heidelberg, Berlin, 1992. MR 93a:30047

[F] O. Forster, Lectures on Riemann Surfaces, Springer-Verlag, New York, Heidelberg, Berlin 1981. MR 83d:3046

[G1] F. P. Gardiner, Approximation of infinite dimensional Teichmüller spaces, Trans. Amer. Math. Soc. 282 (1984), 367-383. MR 85f:30082

[G2] - Teichmüller Theory and Quadratic Differentials, Wiley-Interscience, New York, 1987. MR 88m:32044

[HO] A. Harrington and M. Ortel, The dilatation of an extremal quasi-conformal mapping, Duke Math. J. 43 (1976), 533-544. MR 54:13074

[H] L. A. Harris, Schwarz-Pick systems of pseudometrics for domains in normed linear spaces, Advances in Holomorphy (J. A. Barroso, ed.), North-Holland Mathematics Series 34 North-Holland, Amsterdam, New York, Oxford, 1979. MR 80j:32043

[Kn] M. I. Knopp, A corona theorem for automorphic forms and related results, Amer. J. Math. 91 (1969), 599-618. MR 40:4450

[Ko] C. J. Kolaski, Isometries of Bergman spaces over bounded Runge domains, Canadian J. of Math. 33 (1981), 1157-1164. MR 83b:32028

[Kr] I. Kra, Automorphic Forms and Kleinian Groups, Benjamin, Reading, Massachusetts, 1972. MR 50:10242

[La] N. Lakic, An isometry theorem for quadraric differentials on Riemann surfaces of finite genus, Trans. Amer. Math. Soc., to appear.

[Le] O. Lehto, Univalent Functions and Teichmüller Spaces, Springer-Verlag, New York, Heidelberg, Berlin, 1987. MR 88f:30073

[MM] A. Marden and H. Masur, A foliation of Teichmüller space by twist invariant disks, Math. Scand. 36 (1975), 211-228. MR 52:14393

[Na] S. Nag, The Complex Analytic Theory of Teichmüller Spaces, John Wiley and Sons, New York, 1988. MR 89f:32040

[Ng] K.-F. Ng, On a theorem of Dixmier, Math. Scand. 29 (1971), 279-280. MR 49:3504

[O] T. Ohsawa, On the analytic structure of certain infinite dimensional Teichmüller spaces, preprint.

[Ro] H. Royden, Automorphisms and isometries of Teichmüller space, Advances in the Theory of Riemann Surfaces (L. V. Ahlfors et al., eds.), Ann. Math. Stud. 66, Princeton University Press, 1971, pp. 369-384. MR 46:2037

[Ru1] W. Rudin, Function theory in the unit ball of $\mathbb{C}^{n}$, Springer-Verlag, New York, Heidelberg, Berlin, 1980. MR 82i:32002

[Ru2] - Functional Analysis, Second Edition, McGraw-Hill, New York, 1992.

[SW] A. L. Shields and D. L. Williams, Bounded projections, duality, and multipliers in spaces of analytic functions, Trans. Amer. Math. Soc. 162 (1971), 287-302. MR 44:790

[S1] K. Strebel, On quadratic differentials and extremal quasiconformal mappings, Proceedings Int. Congr. Math. Vancouver 1974, Volume 2, Canadian Mathematical Congress, 1975, pp. 223-227. MR 58:22549

[S2] _ On the existence of extremal Teichmüller mappings, J. Anal. Math. 30 (1976), 464-480. MR 55:12912 
[S3] $\frac{1}{30072}$ Quadratic Differentials, Springer-Verlag, Berlin and New York, 1984. MR 86a: 30072

[T] H. Tanigawa, Holomorphic families of geodesic discs in infinite dimensional Teichmüller spaces, Nagoya Math. J. 127 (1992), 117-128. MR 93i:32027

Department of Mathematics, Cornell University, IthacA, New York 14853

E-mail address: cliff@math.cornell.edu

Department of Mathematics, Brooklyn College, City University of New York, BrookLYN, NEW YORK 11210

E-mail address: fpgbc@cunyvm.cuny.edu 